\title{
ON THE CONSTRUCTION OF REAL NON-SELFADJOINT TRIDIAGONAL MATRICES WITH PRESCRIBED THREE SPECTRA*
}

\author{
WEI-RU XU ${ }^{\dagger}$, NATÁLIA BEBIANO ${ }^{\ddagger}$, AND GUO-LIANG CHEN ${ }^{\S}$
}

\begin{abstract}
Non-selfadjoint tridiagonal matrices play a role in the discretization and truncation of the Schrödinger equation in some extensions of quantum mechanics, a research field particularly active in the last two decades. In this article, we consider an inverse eigenvalue problem that consists of the reconstruction of such a real nonselfadjoint matrix from its prescribed eigenvalues and those of two complementary principal submatrices. Necessary and sufficient conditions under which the problem has a solution are presented, and uniqueness is discussed. The reconstruction is performed by using a modified unsymmetric Lanczos algorithm, designed to solve the proposed inverse eigenvalue problem. Some illustrative numerical examples are given to test the efficiency and feasibility of our reconstruction algorithm.
\end{abstract}

Key words. inverse eigenvalue problem, non-selfadjoint tridiagonal matrix, modified unsymmetric Lanczos algorithm, spectral data

AMS subject classifications. 65F18, 65F15, 15A18, 15A29

1. Introduction. The process of manifesting the dynamical behavior of a system from $a$ priori known physical magnitudes, such as mass, length, elasticity, inductance, and capacitance, is referred to as a direct problem. The problem of determining the physical parameters of the system in terms of its observed, or expected, dynamic behavior is an inverse problem. Both problems are of great importance in applications. The goal of this paper is to study an inverse eigenvalue problem for tridiagonal matrices of the form

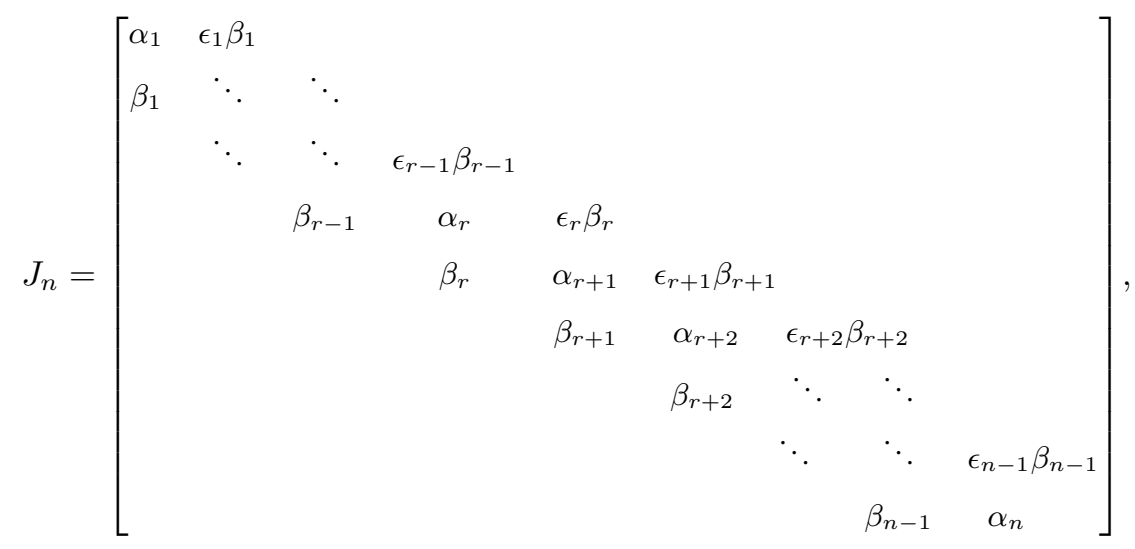

where all the diagonal entries are real, the subdiagonal entries are positive and $\epsilon_{i} \in\{1,-1\}$, for $i=1,2, \ldots, n-1$. These matrices, called pseudo-Jacobi matrices, are related to the selfadjoint involutory matrix $H=\operatorname{diag}\left(\delta_{1}, \delta_{2}, \ldots, \delta_{n}\right)$, with $\delta_{1}=1$ and $\delta_{i}=\prod_{j=1}^{i-1} \epsilon_{j}$, for $i=2, \ldots, n$, as follows: Consider $\mathbb{C}^{n}$ endowed with the indefinite inner product $[\cdot, \cdot]$ defined as $[\mathbf{x}, \mathbf{y}]:=\langle H \mathbf{x}, \mathbf{y}\rangle$ for any $\mathbf{x}, \mathbf{y} \in \mathbb{C}^{n}$, where $\langle\cdot, \cdot\rangle$ is the standard Euclidean inner product.

*Received February 10, 2018. Accepted August 5, 2019. Published online on October 29, 2019. Recommended by Froilan Dopico.

${ }^{\dagger}$ School of Mathematical Sciences, Sichuan Normal University, Chengdu 610066, P. R. China (weiruxu@foxmail.com).

${ }^{\ddagger}$ CMUC, Department of Mathematics, University of Coimbra, P 3001-454 Coimbra, Portugal (bebiano@mat.uc.pt).

${ }^{\S}$ School of Mathematical Sciences, Shanghai Key Laboratory of PMMP, East China Normal University, Shanghai 200241, P. R. China (glchen@math. ecnu. edu. cn). 


\section{ETNA}

Kent State University and

Johann Radon Institute (RICAM)

The $H$-adjoint of a real matrix $A$ is the unique $n \times n$ matrix, written $A^{\#}$, which satisfies

$$
[A \mathbf{x}, \mathbf{y}]=\left[\mathbf{x}, A^{\#} \mathbf{y}\right]
$$

for all $\mathbf{x}, \mathbf{y} \in \mathbb{C}^{n}$. In particular, if $A=A^{\#}$, or equivalently, if $A=H A^{\mathrm{T}} H, A$ is referred to as $H$-symmetric or pseudo-symmetric. Thus, the matrix $J_{n}$ in (1.1) is pseudo-symmetric. If $A^{\#} A=I_{n}, A$ is called $H$-orthogonal or pseudo-orthogonal. Let $\boldsymbol{\beta}=\left(\beta_{1}, \ldots, \beta_{n-1}\right)$, and let $\boldsymbol{\epsilon}=\left(\epsilon_{1}, \ldots, \epsilon_{n-1}\right)$ be the so called sign vector. We denote the set of matrices of the form (1.1) by $\mathcal{J}(n, \boldsymbol{\epsilon}, \boldsymbol{\beta})$. If $\boldsymbol{\epsilon}$ is a vector with all entries equal to one, then $J_{n}$ reduces to a Jacobi matrix.

Pseudo-symmetric matrices usually appear in non-Hermitian quantum mechanics [12], where $H$ is the sign operator (that is, $H^{2}$ is the identity). A sign change in one of the components in $\epsilon$ may lead to strong perturbations in the spectral properties of the matrices in $\mathcal{J}(n, \boldsymbol{\epsilon}, \boldsymbol{\beta})$. The study of pseudo-Jacobi matrices extends the well-known theory of Jacobi matrices, which arise in a variety of applications in different fields such as classical moment problems [1], vibrating systems [15], etc. The discretization and truncation of the Schrödinger equation in non-Hermitian quantum mechanics leads to pseudo-Jacobi matrices [4]. Research on inverse eigenvalue problems for Jacobi matrices originated several fruitful results; see $[2,7,9,10,11,13,14,15,16,17,23,24,25,26,27,28]$ and the references therein. In contrast, the theory concerning the pseudo-Jacobi case constitutes a small part of the literature. The problems in this area deserve attention in order to extend the classical theory of the Jacobi case. At present, some developments focusing on pseudo-Jacobi inverse eigenvalue problem, abbreviated by the acronym PJIEP, have been obtained; see [3, 4, 5, 6, 18, 21, 29, 30]. This paper is in the continuation of this research field. Our work proceeds along the conceptual lines of the standard Jacobi case, but some remarkable differences occur. First, as the matrix $H$ that fixes the inner product is indefinite, there may appear Lanczos vectors with zero norm. Second, the mathematical manipulations are more involved.

In what follows, the principal submatrix of $J_{n}$ in the lines $(\omega, \ldots, v), 1 \leq \omega<v \leq n$, will be denoted by

$$
J_{\omega, v}=\left[\begin{array}{ccccc}
\alpha_{\omega} & \epsilon_{\omega} \beta_{\omega} & & & \\
\beta_{\omega} & \alpha_{\omega+1} & \epsilon_{\omega+1} \beta_{\omega+1} & & \\
& \beta_{\omega+1} & \ddots & \ddots & \\
& & \ddots & \ddots & \epsilon_{v-1} \beta_{v-1} \\
& & & \beta_{v-1} & \alpha_{v}
\end{array}\right],
$$

and $J_{1, v}$ simply by $J_{v}$. We will consider the following inverse problem:

PJIEP. Let a sign vector $\epsilon$ and the sets $\boldsymbol{\lambda}=\left\{\lambda_{i}\right\}_{i=1}^{n} \subset \mathbb{C}, \boldsymbol{\mu}_{1}=\left\{\mu_{i}\right\}_{i=1}^{r} \subset \mathbb{R}$, and $\boldsymbol{\mu}_{2}=\left\{\mu_{i}\right\}_{i=r+1}^{n-1} \subset \mathbb{R}, 1 \leq r \leq n-2$ be given, where $\boldsymbol{\lambda}$ is closed under complex conjugation and the elements of both $\boldsymbol{\mu}_{1}$ and $\boldsymbol{\mu}_{2}$ are pairwise distinct. Construct a pseudo-Jacobi matrix $J_{n} \in \mathcal{J}(n, \boldsymbol{\epsilon}, \boldsymbol{\beta})$ such that $\boldsymbol{\lambda}, \boldsymbol{\mu}_{1}$, and $\boldsymbol{\mu}_{2}$ are, respectively, the spectra of the matrices $J_{n}, J_{r}$, and $J_{r+2, n}$.

Before solving this computability problem, we determine a necessary and sufficient condition under which this problem has a solution. In [18], Mirzaei investigated the particular case of the PJIEP in which the elements of $\boldsymbol{\lambda}, \boldsymbol{\mu}_{1}$, and $\boldsymbol{\mu}_{2}$ are real pairwise distinct numbers and $\boldsymbol{\mu}_{1} \cap \boldsymbol{\mu}_{2}=\varnothing$. The special case in which $H=I_{r} \oplus-I_{1} \oplus I_{n-r-1}$ was solved by $\mathrm{Xu}$, Bebiano, and Chen [29].

This article is organized as follows. In Section 2, we present a modified unsymmetric Lanczos algorithm to construct a matrix $\widehat{J}_{n} \in \mathcal{J}(n, \boldsymbol{\epsilon}, \boldsymbol{\beta})$ whose eigenvalues are real and pairwise distinct. In Section 3, a necessary and sufficient condition under which the PJIEP has 
a solution is stated in the cases when $\boldsymbol{\mu}_{1} \cap \boldsymbol{\mu}_{2}=\varnothing$ and $\boldsymbol{\mu}_{1} \cap \boldsymbol{\mu}_{2} \neq \varnothing$. A numerical algorithm to solve the PJIEP is proposed in Section 4. In Section 5, numerical examples illustrate our approach to the PJIEP and test the efficiency and feasibility of the reconstruction algorithm. In Section 6, some conclusions are drawn. The theoretical results stated in Sections 2 and 3 are proved in the Appendices A and B.

2. Modified unsymmetric Lanczos algorithms. Throughout this paper, let $\chi_{\omega, v}(\lambda)=\operatorname{det}\left(\lambda I_{v-\omega+1}-J_{\omega, v}\right)$ and $\sigma\left(J_{\omega, v}\right)$ be, respectively, the characteristic polynomial and the spectrum of the pseudo-Jacobi matrix $J_{\omega, v}$ in (1.2). For simplicity, we denote $\chi_{1, n}(\lambda)$ as $\chi_{n}(\lambda)$. Let $\widehat{J}_{n} \in \mathcal{J}(n, \boldsymbol{\epsilon}, \boldsymbol{\beta})$ have real and distinct eigenvalues $\widehat{\lambda}_{1}, \widehat{\lambda}_{2}, \ldots, \widehat{\lambda}_{n}$ associated with the real eigenvectors $\boldsymbol{v}_{1}, \boldsymbol{v}_{2}, \ldots, \boldsymbol{v}_{n}$, respectively, and let $\Lambda=\operatorname{diag}\left(\widehat{\lambda}_{1}, \widehat{\lambda}_{2}, \ldots, \widehat{\lambda}_{n}\right)$. It can be easily shown that the eigenvectors $\boldsymbol{v}_{i}, i=1,2, \ldots, n$, may be chosen so that they constitute an $H$-orthonormal basis of $\mathbb{R}^{n}$, i.e., $\left[\boldsymbol{v}_{i}, \boldsymbol{v}_{j}\right]=\delta_{i j} \delta_{i}$, where $\delta_{i j}$ denotes the Kronecker delta. Thus $V=\left[\boldsymbol{v}_{1}, \boldsymbol{v}_{2}, \ldots, \boldsymbol{v}_{n}\right] \in \mathbb{R}^{n \times n}$ is $H$-orthogonal, that is, $V^{\#} V=I_{n}$ with $V^{\#}=H V^{\mathrm{T}} H$.

Before presenting our modified Lanczos formalism, we state an useful extension of the Thompson-McEnteggert-Paige theorem [19].

THEOREM 2.1. The first and last entries of the H-orthonormal eigenvectors of a pseudoJacobi matrix with distinct real eigenvalues are both nonzero.

The unsymmetric Lanczos algorithm in [8] can be used to reconstruct a pseudo-Jacobi matrix $\widehat{J}_{n}$ from its distinct real eigenvalues $\widehat{\lambda}_{1}, \widehat{\lambda}_{2}, \ldots, \widehat{\lambda}_{n}$, the first, or the last, entries of the corresponding $H$-orthonormal eigenvectors $\boldsymbol{v}_{1}, \boldsymbol{v}_{2}, \ldots, \boldsymbol{v}_{n}$, and from its pseudo-norms $\delta_{1}, \delta_{2}, \ldots, \delta_{n}$, where $\delta_{j}:=\left[\boldsymbol{v}_{j}, \boldsymbol{v}_{j}\right]$. It should be noticed that $H$ is indefinite and so the induced inner product lacks positivity. Therefore, it must be analyzed whether the $H$-norms of the computed Lanczos vectors do not vanish. By using the unsymmetric Lanczos algorithm for the matrices $\operatorname{diag}\left(\boldsymbol{\mu}_{1}\right)$ and $\operatorname{diag}\left(\boldsymbol{\mu}_{2}\right)$ with the appropriate starting vectors of order $r$ and $n-r-1$, respectively, the pseudo-Jacobi matrices $J_{r}$ and $J_{r+2, n}$ are obtained. The diagonal entry $\alpha_{r+1}$ of $J_{n}$ results from the trace condition and the neighboring off-diagonals come from the Lanczos procedures.

Firstly, we present the backward modified unsymmetric Lanczos algorithm to recover the matrix $\widehat{J}_{n} \in \mathcal{J}(n, \boldsymbol{\epsilon}, \boldsymbol{\beta})$ initialized from the last entries of its $H$-orthonormal eigenvectors $\boldsymbol{v}_{1}, \boldsymbol{v}_{2}, \ldots, \boldsymbol{v}_{n}$.

THEOREM 2.2. Let $\widehat{\lambda}_{1}, \widehat{\lambda}_{2}, \ldots, \widehat{\lambda}_{n}$ be the real pairwise distinct eigenvalues of $\widehat{J}_{n} \in \mathcal{J}(n, \boldsymbol{\epsilon}, \boldsymbol{\beta})$. Given the last entries $\boldsymbol{v}_{n, 1}, \boldsymbol{v}_{n, 2}, \ldots, \boldsymbol{v}_{n, n}$ of the corresponding H-orthonormal eigenvectors $\boldsymbol{v}_{1}, \boldsymbol{v}_{2}, \ldots, \boldsymbol{v}_{n}$, then $\widehat{J}_{n}$ can be constructed by the backward modified unsymmetric Lanczos algorithm in Algorithm 1.

Next, we give a forward modified unsymmetric Lanczos algorithm, initialized with the entries in the first row of the $H$-orthogonal matrix $V$. The proof of this result is similar to that of Theorem 2.2, and so it is omitted.

THEOREM 2.3. Let $\widehat{J}_{n} \in \mathcal{J}(n, \boldsymbol{\epsilon}, \boldsymbol{\beta})$ have distinct real eigenvalues $\widehat{\lambda}_{1}, \widehat{\lambda}_{2}, \ldots, \widehat{\lambda}_{n}$, and let $\boldsymbol{v}_{11}, \boldsymbol{v}_{12}, \ldots, \boldsymbol{v}_{1, n}$ be the first entries of the corresponding $H$-orthonormal eigenvectors $\boldsymbol{v}_{1}, \boldsymbol{v}_{2}, \ldots, \boldsymbol{v}_{n}$. Then, $\widehat{J}_{n}$ can be constructed by the forward modified unsymmetric Lanczos algorithm in Algorithm 2

REMARK 2.4. By executing Algorithm 2 from Theorem 2.3, we find the matrices $Y=\left[\boldsymbol{Y}_{1}, \boldsymbol{Y}_{2}, \ldots, \boldsymbol{Y}_{n}\right]=V^{\mathrm{T}}$ and $Z=\left[\boldsymbol{Z}_{1}, \boldsymbol{Z}_{2}, \ldots, \boldsymbol{Z}_{n}\right]=V^{\#}$ from the initial vector $\boldsymbol{Y}_{1}=\left(\boldsymbol{v}_{11}, \boldsymbol{v}_{12}, \ldots, \boldsymbol{v}_{1, n}\right)^{\mathrm{T}}$.

3. The construction of the pseudo-Jacobi matrix $\boldsymbol{J}_{\boldsymbol{n}}$ in the PJIEP. Let be given a sign vector $\boldsymbol{\epsilon}$ and the sets $\boldsymbol{\lambda}, \boldsymbol{\mu}_{1}$, and $\boldsymbol{\mu}_{2}$ as in the PJIEP. We construct a solution $J_{n} \in \mathcal{J}(n, \boldsymbol{\epsilon}, \boldsymbol{\beta})$ 

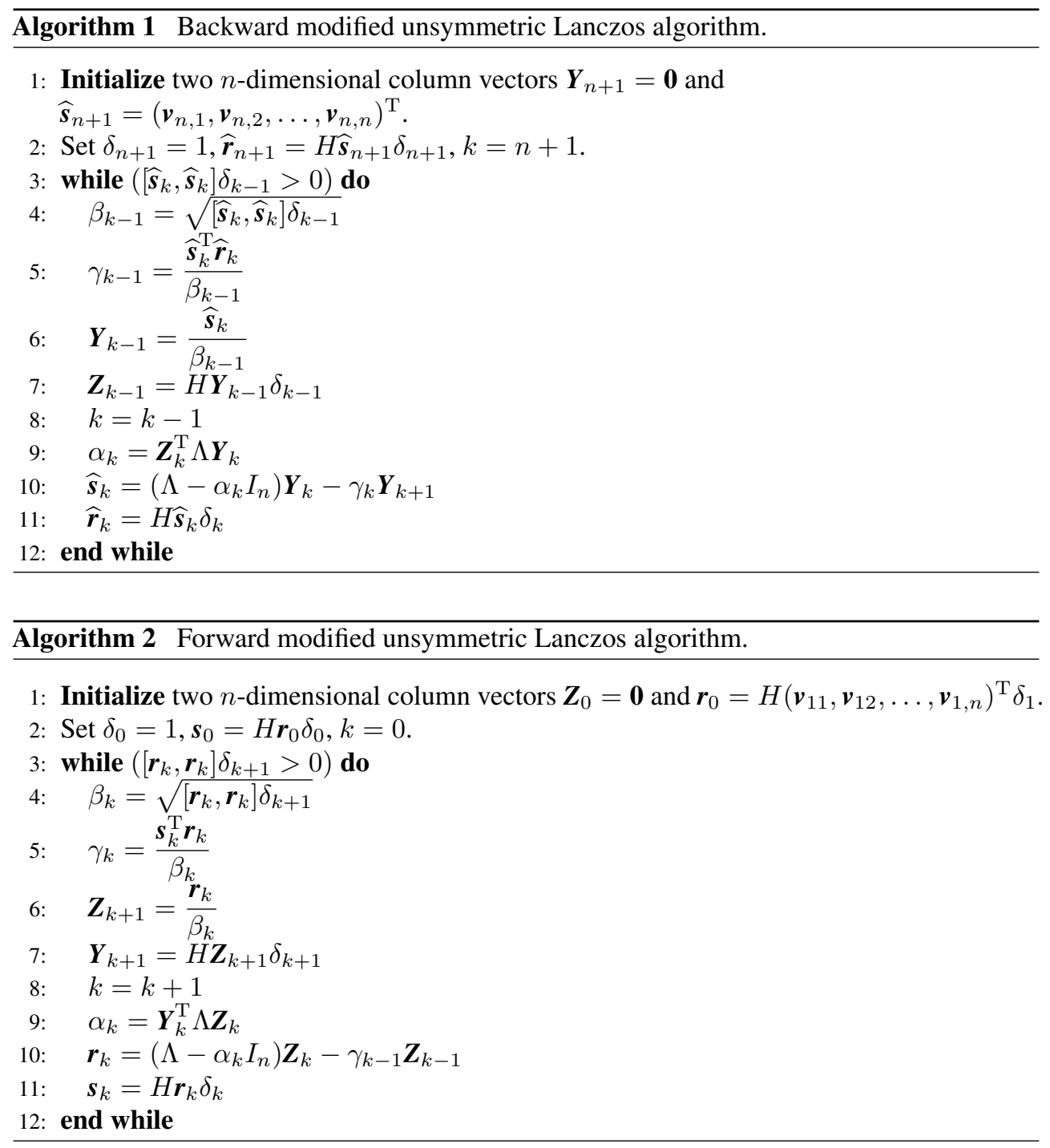

for the PJIEP of the form

$$
J_{n}=\left[\begin{array}{ccc}
J_{r} & \epsilon_{r} \beta_{r} \mathbf{e}_{r} & \mathbf{0} \\
\beta_{r} \mathbf{e}_{r}^{\mathrm{T}} & \alpha_{r+1} & \epsilon_{r+1} \beta_{r+1} \boldsymbol{\omega}_{1}^{\mathrm{T}} \\
\mathbf{0} & \beta_{r+1} \boldsymbol{\omega}_{1} & J_{r+2, n}
\end{array}\right],
$$

where $\mathbf{e}_{r}=(0, \ldots, 0,1)^{\mathrm{T}} \in \mathbb{R}^{r}, \boldsymbol{\omega}_{1}=(1,0, \ldots, 0)^{\mathrm{T}} \in \mathbb{R}^{n-r-1}, \sigma\left(J_{n}\right)=\boldsymbol{\lambda}, \sigma\left(J_{r}\right)=\boldsymbol{\mu}_{1}$, and $\sigma\left(J_{r+2, n}\right)=\boldsymbol{\mu}_{2}$. Hence, the pseudo-Jacobi matrices $J_{r}$ and $J_{r+2, n}$ are, respectively, $H_{1}$-symmetric and $H_{2}$-symmetric for

$$
\begin{aligned}
& H_{1}=\operatorname{diag}\left(1, \epsilon_{1}, \epsilon_{1} \epsilon_{2}, \ldots, \epsilon_{1} \cdots \epsilon_{r-1}\right)=\operatorname{diag}\left(\delta_{1}, \delta_{2}, \ldots, \delta_{r}\right) \quad \text { and } \\
& H_{2}=\operatorname{diag}\left(1, \epsilon_{r+2}, \epsilon_{r+2} \epsilon_{r+3}, \ldots, \epsilon_{r+2} \cdots \epsilon_{n-1}\right)=\operatorname{diag}\left(\frac{\delta_{r+2}}{\delta_{r+2}}, \frac{\delta_{r+3}}{\delta_{r+2}}, \ldots, \frac{\delta_{n}}{\delta_{r+2}}\right) .
\end{aligned}
$$


Let $\mathbf{u}_{i}^{(1)}=\left(u_{1, i}^{(1)}, u_{2, i}^{(1)}, \ldots, u_{r, i}^{(1)}\right)^{\mathrm{T}}$ be the $H_{1}$-orthonormal eigenvector of $J_{r}$ associated with its eigenvalue $\mu_{i}$, for $i=1,2, \ldots, r$, and let $\mathbf{u}_{i}^{(2)}=\left(u_{1, i}^{(2)}, u_{2, i}^{(2)}, \ldots, u_{n-r-1, i}^{(2)}\right)^{\mathrm{T}}$ be the $H_{2}$-orthonormal eigenvector of $J_{r+2, n}$ corresponding to its eigenvalue $\mu_{r+i}$, for $i=1,2, \ldots, n-r-1$. The matrices

$$
\begin{aligned}
U_{1} & =\left[\mathbf{u}_{1}^{(1)}, \mathbf{u}_{2}^{(1)}, \ldots, \mathbf{u}_{r}^{(1)}\right] \quad \text { and } \\
U_{2} & =\left[\mathbf{u}_{1}^{(2)}, \mathbf{u}_{2}^{(2)}, \ldots, \mathbf{u}_{n-r-1}^{(2)}\right]
\end{aligned}
$$

are, respectively, $H_{1}$-orthogonal and $H_{2}$-orthogonal and satisfy

$$
U_{1}^{\#} J_{r} U_{1}=\Lambda_{1}=\operatorname{diag}\left(\boldsymbol{\mu}_{1}\right) \quad \text { and } \quad U_{2}^{\#} J_{r+2, n} U_{2}=\Lambda_{2}=\operatorname{diag}\left(\boldsymbol{\mu}_{2}\right) .
$$

In Theorems 3.1 and 3.2 below, we construct the pseudo-Jacobi matrix $J_{n}$ of the form (3.1) in the following two cases:

$$
\text { (1) } \boldsymbol{\mu}_{1} \cap \boldsymbol{\mu}_{2}=\varnothing \quad \text { and } \quad(2) \boldsymbol{\mu}_{1} \cap \boldsymbol{\mu}_{2} \neq \varnothing .
$$

Although the notation is inevitably heavy, our approach is conceptually simple and consists of the following three main steps.

(i) We recover $J_{r}$ using Algorithm 1 with

$$
H=H_{1}, \quad \Lambda=\Lambda_{1},
$$

and the initializing vector

$$
\widehat{\mathbf{s}}_{r+1}=\left(u_{r, 1}^{(1)}, u_{r, 2}^{(1)}, \ldots, u_{r, r}^{(1)}\right)^{\mathrm{T}} .
$$

Algorithm 1 is used, replacing in steps 1,2 and 10 all the subscripts $n$ by $r$.

(ii) We recover $J_{r+2, n}$ using Algorithm 2 with

$$
H=H_{2}, \quad \Lambda=\Lambda_{2},
$$

and the initializing vector

$$
\boldsymbol{r}_{r+1}=H_{2}\left(u_{11}^{(2)}, u_{12}^{(2)}, \ldots, u_{1, n-r-1}^{(2)}\right)^{\mathrm{T}} \cdot \delta_{r+2} / \delta_{r+2} .
$$

In this forward modified unsymmetric Lanczos algorithm, we replace all the subscripts $n$ and $k$ by $n-r-1$ and $r+k+1$, respectively. Thus, all the $\delta_{r+k+1}$ have a multiplicative factor $1 / \delta_{r+2}$. In step 1, we replace $\boldsymbol{r}_{0}=H\left(\boldsymbol{v}_{11}, \boldsymbol{v}_{12}, \ldots, \boldsymbol{v}_{1, n}\right)^{\mathrm{T}} \delta_{1}$ by $\boldsymbol{r}_{r+1}$. In step 2, we also replace all the subscripts 0 by $r+1$.

(iii) In the backward modified unsymmetric Lanczos process, $Y=\left[\boldsymbol{Y}_{1}, \boldsymbol{Y}_{2}, \ldots, \boldsymbol{Y}_{r}\right]=$ $U_{1}^{\mathrm{T}}$. Similarly, $Z=\left[\boldsymbol{Z}_{1}, \boldsymbol{Z}_{2}, \ldots, \boldsymbol{Z}_{n-r-1}\right]=U_{2}^{\#}$ in the forward modified unsymmetric Lanczos process. In both modified unsymmetric Lanczos processes we have $\gamma_{i}=\epsilon_{i} \beta_{i}$.

THEOREM 3.1. Let be given a sign vector $\epsilon$ and the sets $\lambda, \mu_{1}$, and $\boldsymbol{\mu}_{2}$ as in the PJIEP. Set $x_{j}=-\prod_{i=1}^{n}\left(\lambda_{i}-\mu_{j}\right) \prod_{i=1, i \neq j}^{n-1}\left(\mu_{i}-\mu_{j}\right)^{-1}, j=1,2, \ldots, n-1$. If $\boldsymbol{\mu}_{1} \cap \boldsymbol{\mu}_{2}=\varnothing$, then the PJIEP has a solution if and only if the following conditions are satisfied, and in this case the solution is unique:

(1) $\begin{cases}\delta_{r+1} \delta_{j} x_{j}>0 & \text { if } j=1,2, \ldots, r, \text { and } \\ \delta_{r+1} \delta_{j+1} x_{j}>0 & \text { if } j=r+1, r+2, \ldots, n-1,\end{cases}$

(2) $\epsilon_{r} \sum_{j=1}^{r} x_{j}>0$ and $\epsilon_{r+1} \sum_{j=r+1}^{n-1} x_{j}>0$, 
(3) $\left[\widehat{\boldsymbol{s}}_{k}, \widehat{\boldsymbol{s}}_{k}\right] \delta_{k-1}>0$ for $k=r, r-1, \ldots, 2$, where

$\widehat{\boldsymbol{s}}_{k}=\left(\Lambda_{1}-\alpha_{k} I_{r}\right) \boldsymbol{Y}_{k}-\gamma_{k} \boldsymbol{Y}_{k+1}, \boldsymbol{Y}_{k}=\left(u_{k, 1}^{(1)}, u_{k, 2}^{(1)}, \ldots, u_{k, r}^{(1)}\right)^{\mathrm{T}}$, and $\gamma_{k}=\epsilon_{k} \beta_{k}$,

(4) $\left[\boldsymbol{r}_{r+k+1}, \boldsymbol{r}_{r+k+1}\right] \frac{\delta_{r+k+2}}{\delta_{r+2}}>0$ for $k=1,2, \ldots, n-r-2$, where

$\boldsymbol{r}_{r+k+1}=\left(\Lambda_{2}-\alpha_{r+k+1} I_{n-r-1}\right) \boldsymbol{Z}_{k}-\gamma_{r+k} \boldsymbol{Z}_{k-1}$,

$\boldsymbol{Z}_{k}=H_{2}\left(u_{k, 1}^{(2)}, u_{k, 2}^{(2)}, \ldots, u_{k, n-r-1}^{(2)}\right)^{\mathrm{T}} \cdot \frac{\delta_{r+k+1}}{\delta_{r+2}}$, and

$\gamma_{r+k}=\epsilon_{r+k} \beta_{r+k}$.

THEOREM 3.2. Let be given a sign vector $\epsilon$ and the sets $\boldsymbol{\lambda}, \boldsymbol{\mu}_{1}$, and $\boldsymbol{\mu}_{2}$ as in the PJIEP. Let $\boldsymbol{\mu}_{1} \cap \boldsymbol{\mu}_{2}=\left\{\mu_{i}\right\}_{i=1}^{k}$ and $\mu_{r+i}=\mu_{i}$ for any $i=1,2, \ldots, k$ with $k \leq \min \{r, n-r-1\}$. Assume $\lambda_{i}=\mu_{i}, i=1,2, \ldots, k$, and set

$$
x_{j}=-\prod_{i=k+1}^{n}\left(\lambda_{i}-\mu_{j}\right) \prod_{i=k+1, i \neq j}^{n-1}\left(\mu_{i}-\mu_{j}\right)^{-1}, \quad j=k+1, \ldots, n-1 .
$$

Then, the PJIEP has a solution if and only if

(1) there exist real numbers $\theta_{j} \notin\{0,1\}$ such that

$$
\delta_{r+1} \delta_{j} \theta_{j} x_{r+j}>0 \quad \text { and } \quad \delta_{r+1} \delta_{r+j+1}\left(1-\theta_{j}\right) x_{r+j}>0, \quad \text { for } j=1,2, \ldots, k,
$$

(2) $\begin{cases}\delta_{r+1} \delta_{j} x_{j}>0 & \text { for } j=k+1, \ldots, r, \text { and } \\ \delta_{r+1} \delta_{j+1} x_{j}>0, & \text { for } j=r+k+1, \ldots, n-1,\end{cases}$

(3) $\epsilon_{r}\left(\sum_{j=1}^{k} \theta_{j} x_{r+j}+\sum_{j=k+1}^{r} x_{j}\right)>0$ and

$$
\epsilon_{r+1}\left(\sum_{j=1}^{k}\left(1-\theta_{j}\right) x_{r+j}+\sum_{j=r+k+1}^{n-1} x_{j}\right)>0,
$$

(4) Conditions (3) and (4) in Theorem 3.1 hold.

Furthermore, there are infinite many solutions.

4. Algorithm. Based on Theorems 3.1 and 3.2, we present the following algorithm to construct a pseudo-Jacobi matrix $J_{n}$ in $\mathcal{J}(n, \boldsymbol{\epsilon}, \boldsymbol{\beta})$ that solves the PJIEP. The $\theta_{i}, i=1,2, \ldots, k$, in Theorem 3.2 are randomly selected in $\mathbb{R}-\{0,1\}$ most of the times.

Algorithm 3 A solution of the PJIEP.
Input: $\boldsymbol{\epsilon}, \boldsymbol{\lambda}, \boldsymbol{\mu}_{1}$, and $\boldsymbol{\mu}_{2}$ as in the PJIEP

Output: $J_{n}$

1: if $\boldsymbol{\mu}_{1} \cap \boldsymbol{\mu}_{2}=\varnothing$, then

2: $\quad$ Form

$$
x_{j}=-\frac{\prod_{i=1}^{n}\left(\lambda_{i}-\mu_{j}\right)}{\prod_{i=1, i \neq j}^{n-1}\left(\mu_{i}-\mu_{j}\right)}, \quad j=1,2, \ldots, n-1 .
$$

3: $\quad$ if the conditions (1)-(2) in Theorem 3.1 hold, then

4: $\quad$ Calculate

$$
\beta_{r}:=\left(\epsilon_{r} \sum_{j=1}^{r} x_{j}\right)^{\frac{1}{2}}, \quad \beta_{r+1}:=\left(\epsilon_{r+1} \sum_{j=r+1}^{n-1} x_{j}\right)^{\frac{1}{2}}
$$

5: $\quad$ Compute

$$
\begin{cases}u_{r, j}^{(1)}:=\frac{\sqrt{\delta_{r+1} \delta_{j} x_{j}}}{\beta_{r}}, & j=1,2, \ldots, r, \\ u_{1, j-r}^{(2)}:=\frac{\sqrt{\delta_{r+1} \delta_{j+1} x_{j}}}{\beta_{r+1}}, & j=r+1, r+2, \ldots, n-1 .\end{cases}
$$

6: else 
8: $\quad$ end if

9: else

10: $\quad$ Rearrange the elements in $\boldsymbol{\lambda}, \boldsymbol{\mu}_{1}$, and $\boldsymbol{\mu}_{2}$ such that $\boldsymbol{\mu}_{1} \cap \boldsymbol{\mu}_{2}=\left\{\mu_{i}\right\}_{i=1}^{k}, \mu_{r+i}=\mu_{i}$, and $\lambda_{i}=\mu_{i}$ for $i=1,2, \ldots, k$.

11: $\quad$ Form

$$
x_{j}=-\frac{\prod_{i=k+1}^{n}\left(\lambda_{i}-\mu_{j}\right)}{\prod_{i=k+1, i \neq j}^{n-1}\left(\mu_{i}-\mu_{j}\right)}, \quad j=k+1, \ldots, n-1,
$$

12: $\quad$ Select $\theta_{i}$ in $\mathbb{R}-\{0,1\}$ for all $i=1,2, \ldots, k$.

13: if conditions (1)-(3) in Theorem 3.2 hold, then

14: $\quad$ Calculate

$$
\begin{aligned}
\beta_{r} & :=\left(\epsilon_{r}\left(\sum_{j=1}^{k} \theta_{j} x_{r+j}+\sum_{j=k+1}^{r} x_{j}\right)\right)^{\frac{1}{2}}, \\
\beta_{r+1} & :=\left(\epsilon_{r+1}\left(\sum_{j=1}^{k}\left(1-\theta_{j}\right) x_{r+j}+\sum_{j=r+k+1}^{n-1} x_{j}\right)\right)^{\frac{1}{2}} .
\end{aligned}
$$

15: $\quad$ Compute

$$
u_{r, j}^{(1)}:= \begin{cases}\frac{1}{\beta_{r}} \sqrt{\delta_{r+1} \delta_{j} \theta_{j} x_{r+j}}, & j=1,2, \ldots, k \\ \frac{1}{\beta_{r}} \sqrt{\delta_{r+1} \delta_{j} x_{j}}, & j=k+1, \ldots, r\end{cases}
$$

and

$$
u_{1, j}^{(2)}:= \begin{cases}\frac{1}{\beta_{r+1}} \sqrt{\delta_{r+1} \delta_{r+j+1}\left(1-\theta_{j}\right) x_{r+j}}, & j=1,2, \ldots, k \\ \frac{1}{\beta_{r+1}} \sqrt{\delta_{r+1} \delta_{r+j+1} x_{r+j}}, & j=k+1, \ldots, n-r-1 .\end{cases}
$$

16: else

17: $\quad$ Go to step 12.

18: $\quad$ end if

19: end if

20: if the conditions (3)-(4) in Theorem 3.1 hold, then

21: Construct the pseudo-Jacobi matrix $J_{r}$ from $H_{1}, \boldsymbol{\mu}_{1}$, and $g_{1}=\left(u_{r, 1}^{(1)}, u_{r, 2}^{(1)}, \ldots, u_{r, r}^{(1)}\right)^{\mathrm{T}}$ by Algorithm 1.

22: $\quad$ Compute the pseudo-Jacobi matrix $J_{r+2, n}$ from $H_{2}, \boldsymbol{\mu}_{2}$, and $g_{2}=\left(u_{11}^{(2)}, u_{12}^{(2)}, \ldots, u_{1, n-r-1}^{(2)}\right)^{\mathrm{T}}$

23: else by Algorithm 2.

24: $\quad$ stop

25: end if

26: Compute $\alpha_{r+1}=\sum_{i=1}^{n} \lambda_{i}-\sum_{i=1}^{n-1} \mu_{i}$.

27: return $J_{n}$

Next, we discuss the computational complexity of the above algorithm considering just the case when $\boldsymbol{\mu}_{1} \cap \boldsymbol{\mu}_{2}=\varnothing$.

Step 1 requires $\mathcal{O}(r(n-r-1))$ operations and step $2 \mathcal{O}((n-1)(4 n-5))$. Step 3 requires $\mathcal{O}(2 n)$ operations, step 4 does not require operations, and the cost of step 5 is $\mathcal{O}(n-1)$. As the computational complexities of Algorithms 1 and 2 are both at most $\mathcal{O}\left(15 n^{2}\right)$, the cost of steps $20-22$ is at most $\mathcal{O}\left(15\left(r^{2}+(n-r-1)^{2}\right)\right)$. Finally, step 26 requires $\mathcal{O}(2 n-2)$ operations. Therefore, the total complexity of the algorithm when $\boldsymbol{\mu}_{1} \cap \boldsymbol{\mu}_{2}=\varnothing$ is approximately $\mathcal{O}\left(19 n^{2}+29 r^{2}-29 n r-34 n+29 r+17\right)$. 
5. Numerical experiments. In this section, we present some numerical examples illustrating that Algorithm 3 is theoretically effective to solve the PJIEP. All the tests are performed by using MATLAB R2016a. Because all the pseudo-Jacobi matrices $J_{n}$ in $\mathcal{J}(n, \boldsymbol{\epsilon}, \boldsymbol{\beta})$ rely on the sign vector $\boldsymbol{\epsilon}$, the main diagonal entries $\boldsymbol{\alpha}=\left(\alpha_{1}, \alpha_{2}, \ldots, \alpha_{n}\right)$, and the subdiagonal entries $\boldsymbol{\beta}=\left(\beta_{1}, \beta_{2}, \ldots, \beta_{n-1}\right)$, they can be generated by the following MATLAB code:

$$
\text { diag (alpha) +diag (beta,-1) +diag (beta. *epsilon, } 1 \text { ) . }
$$

Let $\mathrm{i}=\sqrt{-1}$ be the imaginary unit.

EXAMPLE 5.1. Consider an extended harmonic oscillator [12]

$$
H_{\beta}=\frac{\beta}{2}\left(p^{2}+x^{2}\right)+\mathrm{i} \sqrt{2} p, \quad \beta>0,
$$

which acts on $L^{2}$ (the Hilbert space of square integrable differentiable functions of the real variable $x$ ). The operator $p: L^{2} \rightarrow L^{2}$ is the differential operator $f(x) \rightarrow-\mathrm{i} \frac{\mathrm{d} f}{\mathrm{~d} x}$ and $x: L^{2} \rightarrow L^{2}$ is the multiplicative operator $f(x) \rightarrow x f(x)$. With respect to the orthonormal basis constituted by the eigenvectors of the harmonic oscillator, $\phi_{n}(x)=K_{n}\left(x-\frac{\mathrm{d}}{\mathrm{d} x}\right)^{n} \exp \left(-\frac{x^{2}}{2}\right)$ ( $K_{n}$ is the normalization constant), the non-Hermitian operator $H_{\beta}$ is represented by the nonselfadjoint infinite tridiagonal matrix

$$
M_{\beta}=\left[\begin{array}{ccccc}
\frac{1}{2} \beta & -\sqrt{1} & & & \\
\sqrt{1} & \frac{3}{2} \beta & -\sqrt{2} & & \\
& \sqrt{2} & \frac{5}{2} \beta & -\sqrt{3} & \\
& & \sqrt{3} & \frac{7}{2} \beta & \ddots \\
& & & \ddots & \ddots
\end{array}\right]
$$

with real spectrum. The matrix $M_{\beta}$ is pseudo-Hermitian for $H=\operatorname{diag}(1,-1,1,-1, \cdots)$. Then, we consider the finite $r \times r$ tridiagonal matrix

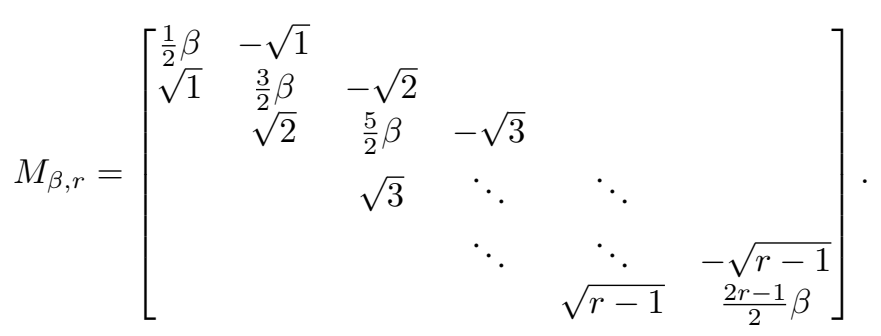

Let a pseudo-Jacobi matrix $J_{n}$ of the form (3.1) be given as follows:

$$
\begin{aligned}
J_{r} & =M_{\beta, r}, & J_{r+2, n} & =M_{\frac{\beta}{2}, r}, \\
\beta_{r} & =\sqrt{r}, & \beta_{r+1} & =\sqrt{r+1}, \\
\alpha_{r+1} & =r, & \epsilon_{r} & =\epsilon_{r+1}=1 .
\end{aligned}
$$

Assume $\boldsymbol{\lambda}=\sigma\left(J_{n}\right), \boldsymbol{\mu}_{1}=\sigma\left(J_{r}\right)$, and $\boldsymbol{\mu}_{2}=\sigma\left(J_{r+2, n}\right)$. We choose the values of $r$ and $\beta$ such that the elements in $\boldsymbol{\mu}_{1}$ and $\boldsymbol{\mu}_{2}$ are all real and pairwise distinct and $\boldsymbol{\mu}_{1} \cap \boldsymbol{\mu}_{2}=\varnothing$.

By Algorithm 3 we can obtain a unique pseudo-Jacobi matrix $\widetilde{J}_{n}$. Then, we compute the spectra $\widetilde{\boldsymbol{\lambda}}, \widetilde{\boldsymbol{\mu}}_{1}$, and $\widetilde{\boldsymbol{\mu}}_{2}$ of $\widetilde{J}_{n}$ and of its principal submatrices $\widetilde{J}_{r}$ and $\widetilde{\widetilde{J}}_{r+2, n}$. In Table 5.1, we present the comparison between $\widetilde{\boldsymbol{\lambda}}, \widetilde{\boldsymbol{\mu}}_{1}, \widetilde{\boldsymbol{\mu}}_{2}, \widetilde{J}_{n}$, and the initial counterparts. If the value of $r$ 
is larger, then the errors $\|\boldsymbol{\lambda}-\widetilde{\boldsymbol{\lambda}}\|_{2},\left\|\boldsymbol{\mu}_{1}-\widetilde{\boldsymbol{\mu}}_{1}\right\|_{2},\left\|\boldsymbol{\mu}_{2}-\widetilde{\boldsymbol{\mu}}_{2}\right\|_{2}$, and $\left\|J_{n}-\widetilde{J}_{n}\right\|_{F}$ will also be larger. Assume that there exist an $H_{1}$-orthogonal matrix $\widetilde{U}_{1}$ and an $H_{2}$-orthogonal matrix $\widetilde{U}_{2}$ such that $\widetilde{U}_{1}^{\#} \widetilde{J}_{r} \widetilde{U}_{1}=\operatorname{diag}\left(\widetilde{\boldsymbol{\mu}}_{1}\right)$ and $\widetilde{U}_{2}^{\#} \widetilde{J}_{r+2, n} \widetilde{U}_{2}=\operatorname{diag}\left(\widetilde{\boldsymbol{\mu}}_{2}\right)$. From the Hoffman-Wielandt theorem for diagonalizable matrices [20], we have that

$$
\begin{aligned}
& \left\|\boldsymbol{\mu}_{1}-\widetilde{\boldsymbol{\mu}}_{1}\right\|_{2} \leq \kappa\left(U_{1}\right) \kappa\left(\widetilde{U}_{1}\right)\left\|J_{r}-\widetilde{J}_{r}\right\|_{F} \quad \text { and } \\
& \left\|\boldsymbol{\mu}_{2}-\widetilde{\boldsymbol{\mu}}_{2}\right\|_{2} \leq \kappa\left(U_{2}\right) \kappa\left(\widetilde{U}_{2}\right)\left\|J_{r+2, n}-\widetilde{J}_{r+2, n}\right\|_{F}
\end{aligned}
$$

by (3.2), where $\kappa(X)$ denotes the condition number of the matrix $X$. Similarly, there exist pseudo-orthogonal matrices $P$ and $\widetilde{P}$ such that $P^{\#} J_{n} P=\operatorname{diag}(\boldsymbol{\lambda})$ and $\widetilde{P}^{\#} \widetilde{J}_{n} \widetilde{P}=\operatorname{diag}(\widetilde{\boldsymbol{\lambda}})$. We also have that

$$
\|\boldsymbol{\lambda}-\tilde{\boldsymbol{\lambda}}\|_{2} \leq \kappa(P) \kappa(\widetilde{P})\left\|J_{n}-\widetilde{J}_{n}\right\|_{F} .
$$

So the large variations in the errors for $J_{n}$ may yield such small variations in the errors for the eigenvalues $\boldsymbol{\lambda}, \boldsymbol{\mu}_{1}$, and $\boldsymbol{\mu}_{2}$ in Table 5.1. Thus, the results agree with our theoretical results established in this paper and demonstrate the feasibility and effectiveness of Algorithm 3.

EXAMPLE 5.2. Let the vector $\boldsymbol{\epsilon}=(1,-1,-1,-1,1,-1,-1,1)$ and the matrix

$$
J_{9}=\left[\begin{array}{rrrrrrrrr}
2 & 2 & 0 & 0 & 0 & 0 & 0 & 0 & 0 \\
2 & -1 & -1 & 0 & 0 & 0 & 0 & 0 & 0 \\
0 & 1 & 3 & -2 & 0 & 0 & 0 & 0 & 0 \\
0 & 0 & 2 & -2 & -2 & 0 & 0 & 0 & 0 \\
0 & 0 & 0 & 2 & 2 & 3 & 0 & 0 & 0 \\
0 & 0 & 0 & 0 & 3 & -4 & -2 & 0 & 0 \\
0 & 0 & 0 & 0 & 0 & 2 & 1 & -\sqrt{2} & 0 \\
0 & 0 & 0 & 0 & 0 & 0 & \sqrt{2} & 3 & \sqrt{2} \\
0 & 0 & 0 & 0 & 0 & 0 & 0 & \sqrt{2} & -3
\end{array}\right]
$$

be given. Consider $r=4$ and let the spectra $\boldsymbol{\lambda}, \boldsymbol{\mu}_{1}, \boldsymbol{\mu}_{2}$ of $J_{9}$ and of its principal submatrices $J_{4}$ and $J_{6,9}$ be as in Table 5.2. Then, $\boldsymbol{\mu}_{1} \cap \boldsymbol{\mu}_{2}=\varnothing, H_{1}=\operatorname{diag}(1,1,-1,1)$, and $H_{2}=\operatorname{diag}(1,-1,1,1)$. By Algorithm 3, we get

$$
\begin{array}{lll}
x_{1}=-0.35726558990817, & x_{2}=-0.66666666666666, & x_{3}=1.99999999999999, \\
x_{4}=-4.97606774342519, & x_{5}=0.71665054819938, & x_{6}=-3.0000000000001, \\
x_{7}=9.00000000000002, & x_{8}=2.28334945180063 .
\end{array}
$$

It is obvious that the conditions (1) and (2) in Theorem 3.1 hold. Thus,

$$
\begin{aligned}
& g_{1}=(0.29885849072269,0.40824829046386,0.70710678118654,1.11535507165041)^{\mathrm{T}}, \\
& g_{2}=(0.28218405108868,0.57735026918963,1.00000000000000,0.50369186477897)^{\mathrm{T}} .
\end{aligned}
$$

Continuing using Algorithm 3, the pseudo-Jacobi matrix $\widetilde{J}_{9} \in \mathcal{J}(9, \boldsymbol{\epsilon}, \widetilde{\boldsymbol{\beta}})$ in Table 5.3 can be obtained.

This matrix is the unique solution of the PJIEP because we have

$$
\left\|J_{9}-\widetilde{J}_{9}\right\|_{F}=1.80047652073822 \mathrm{e}-13 .
$$

Furthermore, we compute the spectra $\widetilde{\boldsymbol{\lambda}}, \widetilde{\boldsymbol{\mu}}_{1}, \widetilde{\boldsymbol{\mu}}_{2}$ of $\widetilde{J}_{9}$ and of its principal submatrices $\widetilde{J}_{4}$ and $\widetilde{J}_{6,9}$. In Figure 5.1 we compare the computed spectra with the original spectra $\boldsymbol{\lambda}, \boldsymbol{\mu}_{1}$, and $\boldsymbol{\mu}_{2}$. 


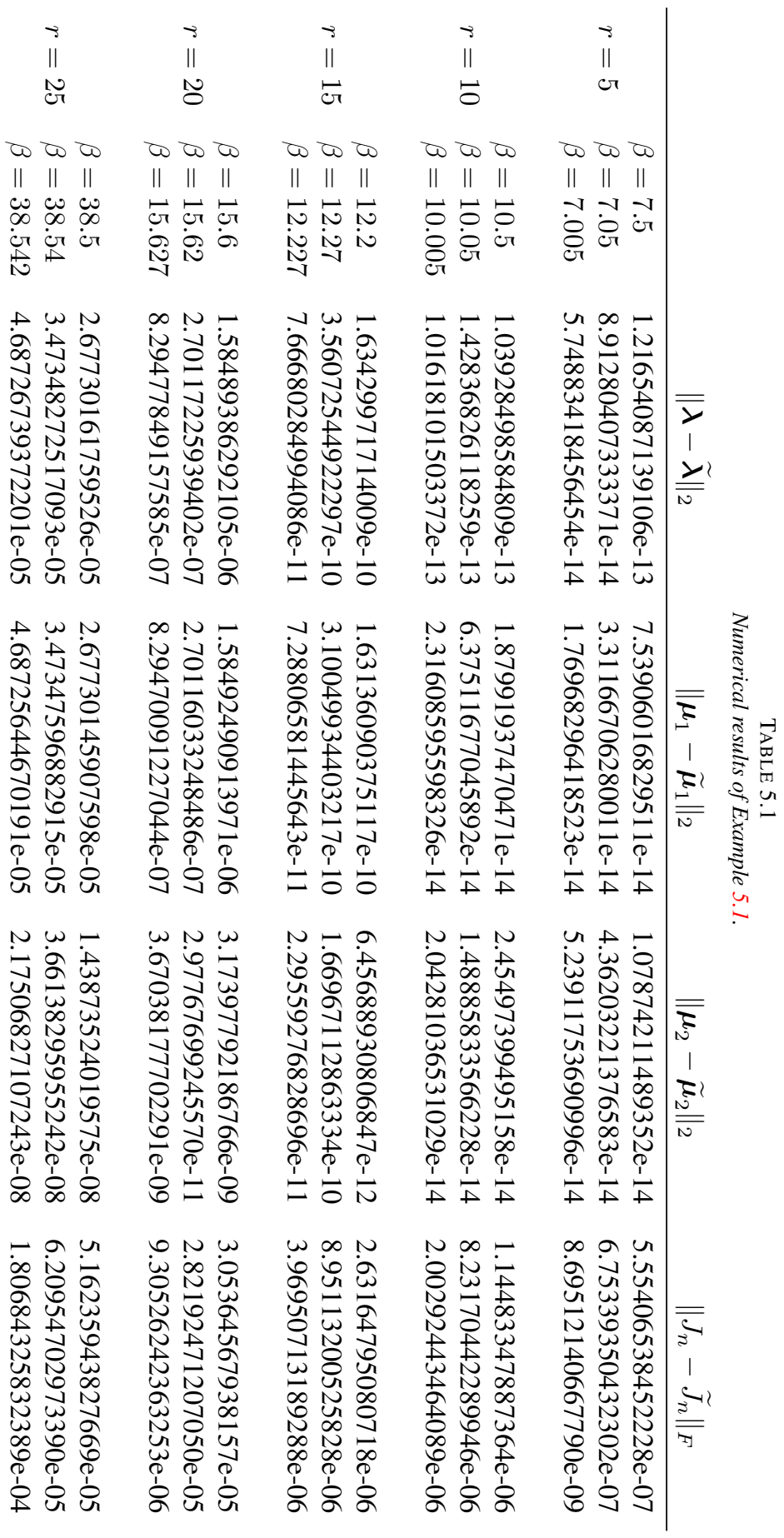


ETNA

Kent State University and

Johann Radon Institute (RICAM)

\begin{tabular}{c|c|c}
\multicolumn{3}{|c}{ TABLE 5.2} \\
The spectra $\boldsymbol{\lambda}=\left\{\lambda_{j}\right\}_{j=1}^{9}, \boldsymbol{\mu}_{1}=\left\{\mu_{j}\right\}_{j=1}^{4}$ and $\boldsymbol{\mu}_{2}=\left\{\mu_{j}\right\}_{j=5}^{8}$. \\
$j$ & $\lambda_{j}$ & $\mu_{j}$ \\
\hline 1 & $2.97935111716971-0.16836804694051 \mathrm{i}$ & 2.73205080756888 \\
2 & $2.97935111716971+0.16836804694051 \mathrm{i}$ & -2.00000000000000 \\
3 & 2.25663440991630 & 2.0000000000000 \\
4 & $1.26336198716348-1.32257216682703 \mathrm{i}$ & -0.73205080756888 \\
5 & $1.26336198716348+1.32257216682703 \mathrm{i}$ & 2.37228132326901 \\
6 & -0.03727254885815 & 1.00000000000000 \\
7 & -1.96441245351364 & -3.00000000000000 \\
8 & -4.46198378686847 & -3.37228132326901 \\
9 & -3.27839182934239 &
\end{tabular}

Main diagonal $\widetilde{\boldsymbol{\alpha}}=\left(\widetilde{\alpha}_{1}, \widetilde{\alpha}_{2}, \ldots, \widetilde{\alpha}_{9}\right)$ and subdiagonal $\widetilde{\boldsymbol{\beta}}=\left(\widetilde{\beta}_{1}, \widetilde{\beta}_{2}, \ldots, \widetilde{\beta}_{8}\right)$ of $\widetilde{J}_{9} \in \mathcal{J}(9, \boldsymbol{\epsilon}, \widetilde{\boldsymbol{\beta}})$.

\begin{tabular}{c|c|c}
$j$ & $\widetilde{\alpha}_{j}$ & $\widetilde{\beta}_{j}$ \\
\hline 1 & 1.99999999999997 & 2.00000000000004 \\
2 & -0.99999999999996 & 1.00000000000001 \\
3 & 2.99999999999997 & 1.99999999999997 \\
4 & -1.99999999999997 & 2.00000000000001 \\
5 & 2.00000000000001 & 3.00000000000001 \\
6 & -3.99999999999999 & 1.99999999999998 \\
7 & 0.99999999999994 & 1.41421356237315 \\
8 & 3.00000000000007 & 1.41421356237305 \\
9 & -3.00000000000002 &
\end{tabular}

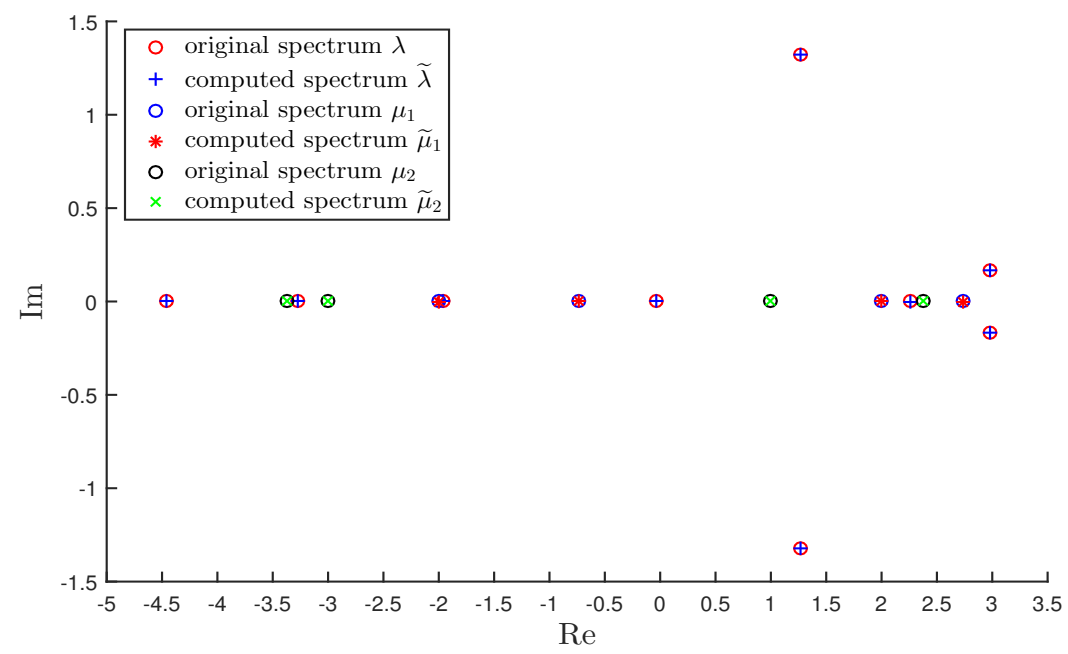

FIG. 5.1. Comparison between the original spectra $\boldsymbol{\lambda}, \boldsymbol{\mu}_{1}, \boldsymbol{\mu}_{2}$ and the computed spectra $\tilde{\boldsymbol{\lambda}}, \widetilde{\boldsymbol{\mu}}_{1}, \widetilde{\boldsymbol{\mu}}_{2}$. 
TABLE 5.4

\begin{tabular}{|c|c|c|}
\hline$\|\boldsymbol{\lambda}-\tilde{\boldsymbol{\lambda}}\|_{2}$ & $\left\|\boldsymbol{\mu}_{1}-\widetilde{\boldsymbol{\mu}}_{1}\right\|_{2}$ & $\left\|\boldsymbol{\mu}_{2}-\widetilde{\boldsymbol{\mu}}_{2}\right\|_{2}$ \\
\hline $6.27822826864024 \mathrm{e}-15$ & $5.61843057806044 \mathrm{e}-15$ & $7.93168690704416 \mathrm{e}-15$ \\
\hline
\end{tabular}

\begin{tabular}{c|c|c}
\multicolumn{3}{c}{ TABLE 5.5 } \\
\multicolumn{3}{|c}{ The spectra $\boldsymbol{\lambda}=\left\{\lambda_{j}\right\}_{j=1}^{9}, \boldsymbol{\mu}_{1}=\left\{\mu_{j}\right\}_{j=1}^{4}$ and $\boldsymbol{\mu}_{2}=\left\{\mu_{j}\right\}_{j=5}^{8}}$. \\
$j$ & $\lambda_{j}$ & $\mu_{j}$ \\
\hline 1 & 2.00000000000000 & 2.00000000000000 \\
2 & -2.00000000000000 & -2.00000000000000 \\
3 & 6.58533601596052 & 2.73205080756888 \\
4 & 3.40647425562398 & -0.73205080756888 \\
5 & 1.79809235457054 & 2.00000000000000 \\
6 & $-1.96971063765212+2.86959352986032 \mathrm{i}$ & -2.00000000000000 \\
7 & $-1.96971063765212-2.86959352986032 \mathrm{i}$ & 3.37228132326901 \\
8 & -0.57578829818534 & -2.37228132326901 \\
9 & -2.27469305266548 &
\end{tabular}

In Table 5.4 we also present their respective absolute errors. The computed spectra are in total accordance with the original spectra within the machine precision.

EXAMPLE 5.3. Let $\boldsymbol{\epsilon}=(-1,-1,1,1,-1,-1,-1,1), r=4$, and

$$
J_{9}=\left[\begin{array}{rrrrrrrrr}
-2 & -2 & 0 & 0 & 0 & 0 & 0 & 0 & 0 \\
2 & 3 & -1 & 0 & 0 & 0 & 0 & 0 & 0 \\
0 & 1 & -1 & 2 & 0 & 0 & 0 & 0 & 0 \\
0 & 0 & 2 & 2 & 5 & 0 & 0 & 0 & 0 \\
0 & 0 & 0 & 5 & 2 & -4 & 0 & 0 & 0 \\
0 & 0 & 0 & 0 & 4 & -3 & -2 & 0 & 0 \\
0 & 0 & 0 & 0 & 0 & 2 & 2 & -\sqrt{2} & 0 \\
0 & 0 & 0 & 0 & 0 & 0 & \sqrt{2} & 4 & \sqrt{2} \\
0 & 0 & 0 & 0 & 0 & 0 & 0 & \sqrt{2} & -2
\end{array}\right] .
$$

Then, $H_{1}=H_{2}=\operatorname{diag}(1,-1,1,1)$, and the spectra $\boldsymbol{\lambda}, \boldsymbol{\mu}_{1}$, and $\boldsymbol{\mu}_{2}$ of $J_{9}$ and of its principal submatrices $J_{4}$ and $J_{6,9}$ are given in Table 5.5.

Obviously, $\boldsymbol{\mu}_{1} \cap \boldsymbol{\mu}_{2}=\{2.00000000000000,-2.00000000000000\}$ and $k=2$. In Algorithm 3, we consider three of the solutions. Firstly, by selecting $\theta_{1}=2$ and $\theta_{2}=-7$, a pseudo-Jacobi matrix $\widetilde{J}_{9}^{(1)} \in \mathcal{J}\left(9, \boldsymbol{\epsilon}, \widetilde{\boldsymbol{\beta}}^{(1)}\right)$ is obtained in Table 5.6. Then, by choosing $\theta_{1}=3$ and $\theta_{2}=-8$, we get another pseudo-Jacobi matrix $\widetilde{J}_{9}^{(2)} \in \mathcal{J}\left(9, \boldsymbol{\epsilon}, \widetilde{\boldsymbol{\beta}}^{(2)}\right)$ whose entries are displayed in Table 5.7. Next, taking $\theta_{1}=5$ and $\theta_{2}=-8$, a pseudo-Jacobi matrix $\widetilde{J}_{9}^{(3)} \in \mathcal{J}\left(9, \boldsymbol{\epsilon}, \widetilde{\boldsymbol{\beta}}^{(3)}\right)$ is obtained and given in Table 5.8.

Finally, we compute the spectra $\widetilde{\boldsymbol{\lambda}}^{(i)}, \widetilde{\boldsymbol{\mu}}_{1}^{(i)}$, and $\widetilde{\boldsymbol{\mu}}_{2}^{(i)}$ of the pseudo-Jacobi matrices $\widetilde{J}_{9}^{(i)}$ and of their principal submatrices $\widetilde{J}_{4}^{(i)}$ and $\widetilde{J}_{6,9}^{(i)}$, for $i=1,2,3$. Comparing these spectra with the original spectra $\boldsymbol{\lambda}, \boldsymbol{\mu}_{1}$, and $\boldsymbol{\mu}_{2}$, Figures 5.2, 5.3, 5.4, and Table 5.9 illustrate that the computed spectra agree with the original spectra up to the machine precision. All the numerical results are in accordance with the theory developed in this paper.

6. Conclusions. In this paper, an inverse eigenvalue problem for Jacobi matrices that was investigated in [17] has been considered in the non-selfadjoint setting. This problem, 
ETNA

Kent State University and

Johann Radon Institute (RICAM)

CONSTRUCTION OF REAL NON-SELFADJOINT TRIDIAGONAL MATRICES

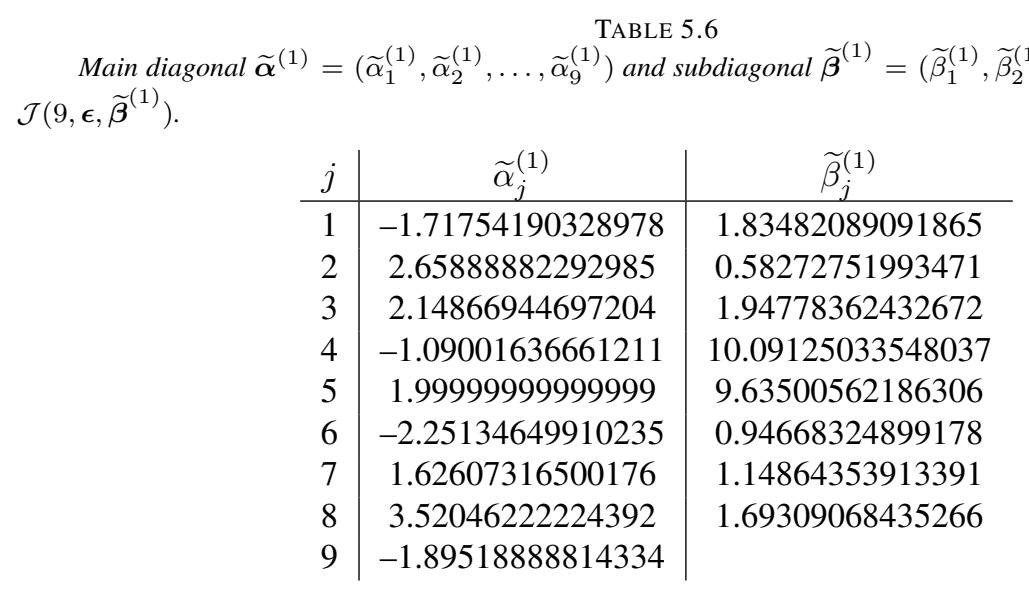

TABLE 5.7

\begin{tabular}{|c|c|c|}
\hline$j$ & $\widetilde{\alpha}_{j}^{(2)}$ & $\widetilde{\beta}_{j}^{(2)}$ \\
\hline 1 & -1.20263604281617 & 1.12069676849459 \\
\hline 2 & 1.81727913229512 & 0.97735873260376 \\
\hline 3 & 2.78441794338492 & 1.72672967266801 \\
\hline 4 & -1.39906103286386 & 10.31988372027510 \\
\hline 5 & 1.99999999999999 & 9.87420882906570 \\
\hline 6 & -2.53333333333336 & 1.50122457136676 \\
\hline 7 & 2.27605177993530 & 0.64677836372989 \\
\hline 8 & 3.00807778349232 & 1.82098682825439 \\
\hline 9 & -1.75079623009426 & \\
\hline
\end{tabular}

Main diagonal $\widetilde{\boldsymbol{\alpha}}^{(3)}=\left(\widetilde{\alpha}_{1}^{(3)}, \widetilde{\alpha}_{2}^{(3)}, \ldots, \widetilde{\alpha}_{9}^{(3)}\right)$ and subdiagonal $\widetilde{\boldsymbol{\beta}}^{(3)}=\left(\widetilde{\beta}_{1}^{(3)}, \widetilde{\beta}_{2}^{(3)}, \ldots, \widetilde{\beta}_{8}^{(3)}\right)$ of $\widetilde{J}_{9}^{(3)} \in$ $\mathcal{J}\left(9, \boldsymbol{\epsilon}, \widetilde{\boldsymbol{\beta}}^{(3)}\right)$.

\begin{tabular}{c|c|c}
$j$ & $\widetilde{\alpha}_{j}^{(3)}$ & $\widetilde{\beta}_{j}^{(3)}$ \\
\hline 1 & -0.88332250873279 & 0.45831627309447 \\
2 & -1.02180790112723 & 3.59721086479957 \\
3 & 5.83279767929948 & 1.17008113254617 \\
4 & -1.92766726943946 & 9.60034721594304 \\
5 & 1.99999999999999 & 9.11957601353623 \\
6 & -3.31462925851710 & 2.60660360466998 \\
7 & 3.19245936689820 & 0.38865718165124 \\
8 & 2.80498308965325 & 1.87250089319368 \\
9 & -1.68281319803435 &
\end{tabular}

TABLE 5.9

Absolute errors between the original spectra $\boldsymbol{\lambda}, \boldsymbol{\mu}_{1}, \boldsymbol{\mu}_{2}$ and the computed spectra $\widetilde{\boldsymbol{\lambda}}^{(i)}, \widetilde{\boldsymbol{\mu}}_{1}^{(i)}, \widetilde{\boldsymbol{\mu}}_{2}^{(i)}$.

\begin{tabular}{c|c|c|c}
$i$ & $\left\|\boldsymbol{\lambda}-\widetilde{\boldsymbol{\lambda}}^{(i)}\right\|_{2}$ & $\left\|\boldsymbol{\mu}_{1}-\widetilde{\boldsymbol{\mu}}_{1}^{(i)}\right\|_{2}$ & $\left\|\boldsymbol{\mu}_{2}-\widetilde{\boldsymbol{\mu}}_{2}^{(i)}\right\|_{2}$ \\
\hline 1 & $2.76195696656855 \mathrm{e}-14$ & $1.39393795312866 \mathrm{e}-14$ & $7.02517689335219 \mathrm{e}-15$ \\
2 & $7.85613248788838 \mathrm{e}-14$ & $2.44249065417534 \mathrm{e}-15$ & $2.97904098389673 \mathrm{e}-15$ \\
3 & $9.20595358801062 \mathrm{e}-13$ & $2.13859186672314 \mathrm{e}-13$ & $5.82417538579527 \mathrm{e}-15$
\end{tabular}




\section{ETNA}

Kent State University and

Johann Radon Institute (RICAM)

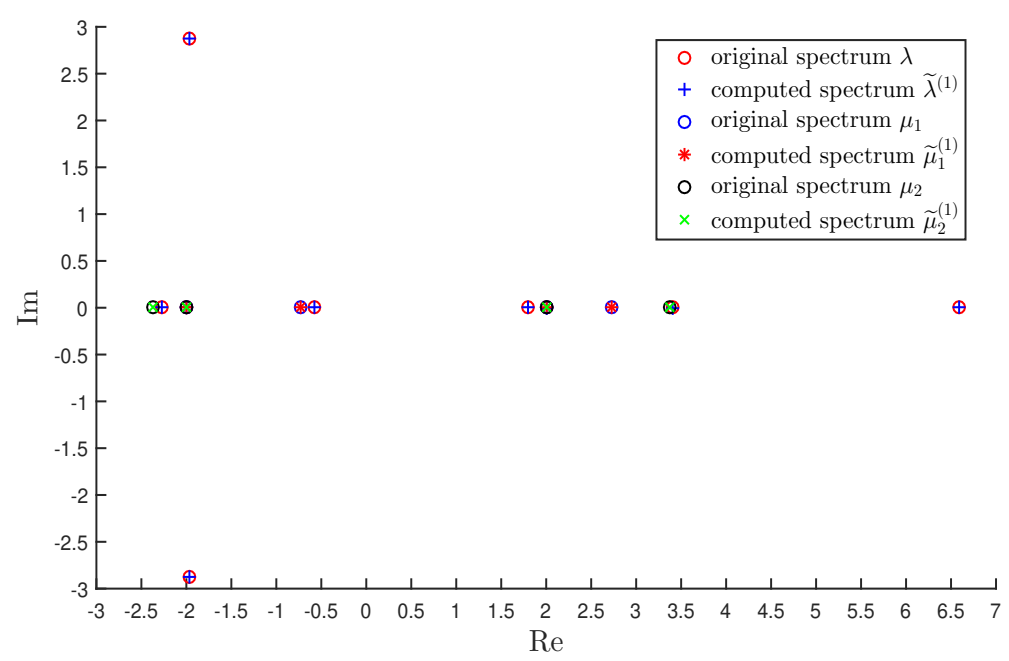

FIG. 5.2. Comparison between the original spectra $\boldsymbol{\lambda}, \boldsymbol{\mu}_{1}, \boldsymbol{\mu}_{2}$ and the computed spectra $\widetilde{\boldsymbol{\lambda}}^{(1)}, \widetilde{\boldsymbol{\mu}}_{1}^{(1)}, \widetilde{\boldsymbol{\mu}}_{2}^{(1)}$.

abbreviated as PJIEP, has been solved from the knowledge of a given sign vector $\epsilon$ and from the prescribed spectra $\boldsymbol{\lambda}, \boldsymbol{\mu}_{1}$, and $\boldsymbol{\mu}_{2}$ of $J_{n}$ and of two complementary principal submatrices, where $\boldsymbol{\lambda}$ is closed under complex conjugation and all the elements in $\boldsymbol{\mu}_{1}$ and $\boldsymbol{\mu}_{2}$ are real pairwise distinct. Necessary and sufficient conditions for the existence of the solution have been found according to the two cases $\boldsymbol{\mu}_{1} \cap \boldsymbol{\mu}_{2}=\varnothing$ and $\boldsymbol{\mu}_{1} \cap \boldsymbol{\mu}_{2} \neq \varnothing$. Then, the desired pseudo-Jacobi matrices have been constructed with the aid of a modified unsymmetric Lanczos algorithm. Furthermore, numerical experiments illustrate the efficiency and feasibility of the proposed construction algorithm (Algorithm 3). Our results extend the previous results obtained in [18] for the unique case when $\boldsymbol{\mu}_{1} \cap \boldsymbol{\mu}_{2}=\varnothing$ as well as in [6] and [29] for the cases $H=I_{r} \oplus-I_{n-r}$ and $H=I_{r} \oplus-I_{1} \oplus I_{n-r-1}$, respectively. If the sets $\boldsymbol{\mu}_{1}$ and $\boldsymbol{\mu}_{2}$ in the PJIEP have complex elements and are closed under conjugation, then the present approach does not apply. This is an open problem that deserves future attention.

Acknowledgments. The authors are grateful to the referees for most valuable comments. They also thank Professor F. Dopico for carefully reading the manuscript and for his helpful and pertinent suggestions. This work was supported by the National Natural Science Foundation of China (Grant No. 11471122), and in part by the Science and Technology Commission of Shanghai Municipality (Grant No. 18dz2271000). The first author was also partially supported by the Natural Science Foundation of Shandong Province (Grant No. ZR2017MA050). The second author acknowledges financial support from the Centre for Mathematics of University of Coimbra (funded by the Portuguese Government through FCT/MEC and by European RDF through Partnership Agreement PT2020).

Appendix A. Proofs of Theorems 2.1 and 2.2. The proofs of the theorems in Section 2 require the following lemmas.

LEMma A.1. Let $\widehat{J}_{n} \in \mathcal{J}(n, \boldsymbol{\epsilon}, \boldsymbol{\beta})$ have distinct real eigenvalues $\widehat{\lambda}_{1}, \widehat{\lambda}_{2}, \ldots, \widehat{\lambda}_{n}$. Then, the adjugate of $\widehat{\lambda}_{j} I_{n}-\widehat{J}_{n}$ is

$$
\operatorname{adj}\left(\widehat{\lambda}_{j} I_{n}-\widehat{J}_{n}\right)=\chi_{n}^{\prime}\left(\widehat{\lambda}_{j}\right) \boldsymbol{v}_{j} \boldsymbol{v}_{j}^{\mathrm{T}} H \delta_{j},
$$




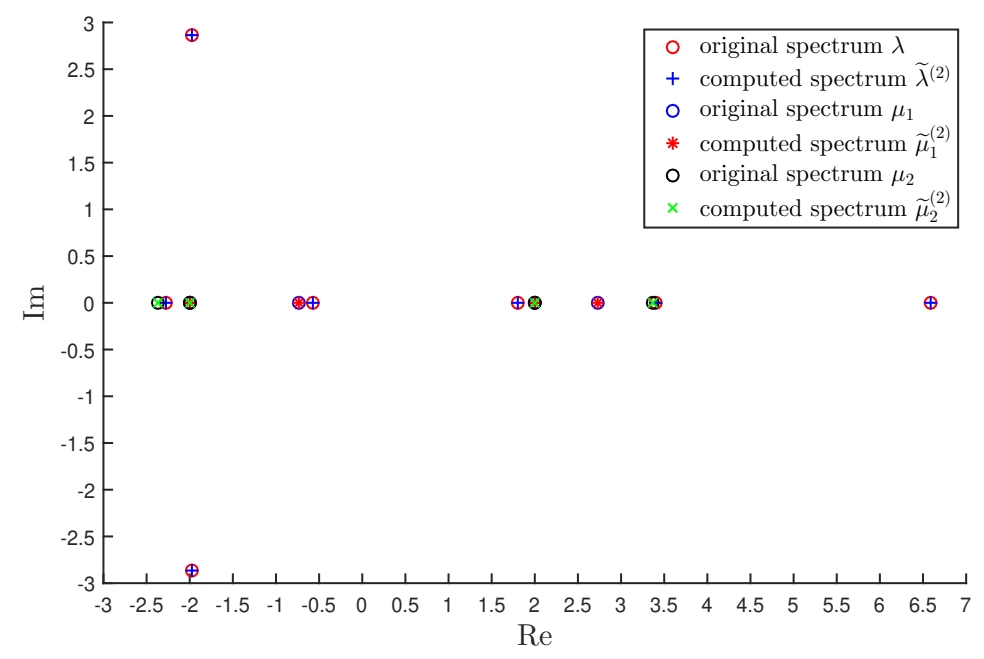

FIG. 5.3. Comparison between the original spectra $\boldsymbol{\lambda}, \boldsymbol{\mu}_{1}, \boldsymbol{\mu}_{2}$ and the computed spectra $\widetilde{\boldsymbol{\lambda}}^{(2)}, \widetilde{\boldsymbol{\mu}}_{1}^{(2)}, \widetilde{\boldsymbol{\mu}}_{2}^{(2)}$.

where $\boldsymbol{v}_{j}$ is the jth column of an H-orthogonal diagonalizing matrix of $\widehat{J}_{n}$ and where $\chi_{n}^{\prime}\left(\widehat{\lambda}_{j}\right)=\prod_{1=i \neq j}^{n}\left(\widehat{\lambda}_{j}-\widehat{\lambda}_{i}\right)$.

Proof. Under the hypothesis on $\widehat{J}_{n}$, there exists an $H$-orthogonal matrix $V$ such that

$$
\widehat{J}_{n} V=V \Lambda, \quad \Lambda=\operatorname{diag}\left(\widehat{\lambda}_{1}, \widehat{\lambda}_{2}, \ldots, \widehat{\lambda}_{n}\right) .
$$

Thus,

$$
\begin{aligned}
\operatorname{adj}\left(\lambda I_{n}-\widehat{J}_{n}\right) & =\operatorname{det}\left(\lambda I_{n}-\widehat{J}_{n}\right) \cdot\left(\lambda I_{n}-\widehat{J}_{n}\right)^{-1}=\operatorname{det}\left(\lambda I_{n}-\widehat{J}_{n}\right) \cdot V\left(\lambda I_{n}-\Lambda\right)^{-1} V^{\#} \\
& =\sum_{i=1}^{n} \frac{\operatorname{det}\left(\lambda I_{n}-\widehat{J}_{n}\right)}{\lambda-\widehat{\lambda}_{i}} \boldsymbol{v}_{i} \boldsymbol{v}_{i}^{\mathrm{T}} H \delta_{i} .
\end{aligned}
$$

From the last equality, we easily obtain (A.1).

REMARK A.2. Matrices in $\mathcal{J}(n, \boldsymbol{\epsilon}, \boldsymbol{\beta})$ may exist with multiple eigenvalues (see Examples 4.1 and 4.2 in [29]). It has been shown in Lemma A.1 that, if $\widehat{J}_{n} \in \mathcal{J}(n, \boldsymbol{\epsilon}, \boldsymbol{\beta})$ is diagonalizable and has a multiple eigenvalue $\widehat{\lambda}_{j}$, then the equality (A.1) also holds.

LEMMA A.3. Under the assumptions in Lemma A.1, both of the following statements hold:

(1) If $\omega \leq v$, then $\chi_{1, \omega-1}\left(\widehat{\lambda}_{j}\right) \beta_{\omega} \cdots \beta_{v-1} \chi_{v+1, n}\left(\widehat{\lambda}_{j}\right)=\chi_{n}^{\prime}\left(\widehat{\lambda}_{j}\right) \boldsymbol{v}_{\omega j} \boldsymbol{v}_{v j} \delta_{\omega} \delta_{j}$;

(2) If $\omega \geq v$, then $\chi_{1, v-1}\left(\widehat{\lambda}_{j}\right) \beta_{v} \cdots \beta_{\omega-1} \chi_{\omega+1, n}\left(\widehat{\lambda}_{j}\right)=\chi_{n}^{\prime}\left(\widehat{\lambda}_{j}\right) \boldsymbol{v}_{\omega j} \boldsymbol{v}_{v j} \delta_{v} \delta_{j}$, where $\boldsymbol{v}_{i j}$ is the ith component of $\boldsymbol{v}_{j}$.

Proof. If $\omega \leq v$, we consider the $(\omega, v)$ th entry on both sides of the equality (A.1), and we get

$$
\chi_{1, \omega-1}\left(\widehat{\lambda}_{j}\right) \beta_{\omega} \cdots \beta_{v-1} \epsilon_{\omega} \cdots \epsilon_{v-1} \chi_{v+1, n}\left(\widehat{\lambda}_{j}\right)=\chi_{n}^{\prime}\left(\widehat{\lambda}_{j}\right) \boldsymbol{v}_{\omega j} \boldsymbol{v}_{v j} \delta_{v} \delta_{j} .
$$

Then, (1) holds because $\epsilon_{\omega} \cdots \epsilon_{v-1}=\frac{\delta_{v}}{\delta_{\omega}}$.

If $\omega \geq v$, we take the $(\omega, v)$ th entry on both sides of the equality (A.1). Then,

$$
\chi_{1, v-1}\left(\widehat{\lambda}_{j}\right) \beta_{v} \cdots \beta_{\omega-1} \chi_{\omega+1, n}\left(\widehat{\lambda}_{j}\right)=\chi_{n}^{\prime}\left(\widehat{\lambda}_{j}\right) \boldsymbol{v}_{\omega j} \boldsymbol{v}_{v j} \delta_{v} \delta_{j},
$$




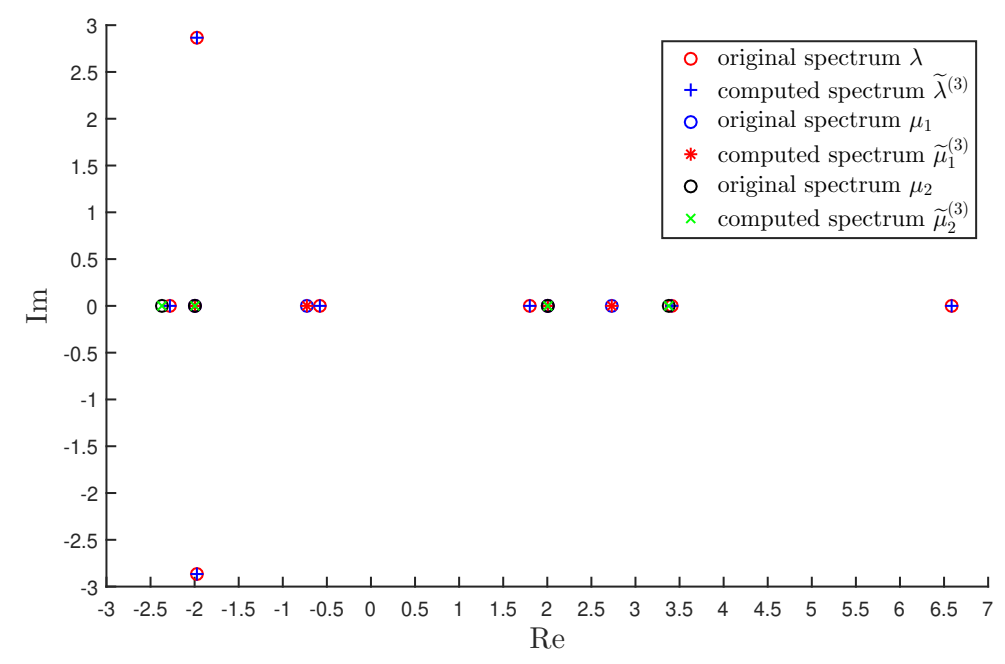

FIG. 5.4. Comparison between the original spectra $\boldsymbol{\lambda}, \boldsymbol{\mu}_{1}, \boldsymbol{\mu}_{2}$ and the computed spectra $\widetilde{\boldsymbol{\lambda}}^{(3)}, \widetilde{\boldsymbol{\mu}}_{1}^{(3)}, \widetilde{\boldsymbol{\mu}}_{2}^{(3)}$.

and (2) follows.

When $(\omega, v)$ is, respectively, taken to be $(1, n),(n, 1),(1,1)$, and $(n, n)$, the following immediate consequence of Lemma A.3 holds.

LEMMA A.4. Under the assumptions in Lemma A.1, we have

(1) $\chi_{n}^{\prime}\left(\widehat{\lambda}_{j}\right) \boldsymbol{v}_{1 j} \boldsymbol{v}_{n j}=\delta_{1} \delta_{j} \beta_{1} \beta_{2} \cdots \beta_{n-1}$;

(2) $\chi_{n}^{\prime}\left(\widehat{\lambda}_{j}\right) v_{1 j}^{2}=\delta_{1} \delta_{j} \chi_{2, n}\left(\widehat{\lambda}_{j}\right)$;

(3) $\chi_{n}^{\prime}\left(\widehat{\lambda}_{j}\right) v_{n j}^{2}=\delta_{n} \delta_{j} \chi_{1, n-1}\left(\widehat{\lambda}_{j}\right)$.

Proof of Theorem 2.1. The first item in the above lemma was obtained in [5, Lemma 2.2]. Because $0 \neq \chi_{n}^{\prime}\left(\widehat{\lambda}_{j}\right) \in \mathbb{R}$, from (1) it follows that $0 \neq \boldsymbol{v}_{1 j} \boldsymbol{v}_{n j} \in \mathbb{R}$.

Proof of Theorem 2.2. From Lemma A.4 (1) it follows that $\beta_{1} \beta_{2} \cdots \beta_{n-1} \neq 0$ and $0 \neq \boldsymbol{v}_{n, i} \in \mathbb{R}$, for $i=1,2, \ldots, n$. Since $\left(\boldsymbol{v}_{n, 1}, \boldsymbol{v}_{n, 2}, \ldots, \boldsymbol{v}_{n, n}\right)$ is the last row of the $H$ orthogonal matrix $V$, it follows that

$$
\delta_{1} \boldsymbol{v}_{n, 1}^{2}+\delta_{2} \boldsymbol{v}_{n, 2}^{2}+\cdots+\delta_{n} \boldsymbol{v}_{n, n}^{2}=\delta_{n}
$$

Assume that $\left[\widehat{\boldsymbol{s}}_{k}, \widehat{\boldsymbol{s}}_{k}\right] \delta_{k-1}>0$, for $k=n, n-1, \ldots, 2$, and also $\left[\widehat{\boldsymbol{s}}_{n+1}, \widehat{\boldsymbol{s}}_{n+1}\right] \delta_{n}>0$. We first show that $\boldsymbol{Y}_{n}, \boldsymbol{Y}_{n-1}, \ldots, \boldsymbol{Y}_{1}$, computed by this algorithm, are the columns of the $H$-orthogonal matrix $Y=\left[\boldsymbol{Y}_{1}, \boldsymbol{Y}_{2}, \ldots, \boldsymbol{Y}_{n}\right]$, and we demonstrate that the following pseudoorthogonality relations hold:

$$
\left[\boldsymbol{Y}_{i}, \boldsymbol{Y}_{j}\right]=\delta_{i j} \delta_{i}, \quad \text { for } j=n, n-1, \ldots, i \text { and } i=n, n-1, \ldots, 1
$$

From (A.2) and steps 4 and 6, (A.3) follows for $i=n$. If (A.3) holds for $i=n, n-1, \ldots, l$, we then prove that it also holds for $i=l-1$.

If $j=l-1$, steps 4 and 6 imply that (A.3) holds because

$$
\left[\boldsymbol{Y}_{l-1}, \boldsymbol{Y}_{l-1}\right]=\frac{1}{\beta_{l-1}^{2}}\left[\widehat{\boldsymbol{s}}_{l}, \widehat{\boldsymbol{s}}_{l}\right]=\delta_{l-1}
$$


For $j \geq l$, from steps 6 and 10 we have

$$
\begin{aligned}
{\left[\boldsymbol{Y}_{l-1}, \boldsymbol{Y}_{j}\right] } & =\frac{1}{\beta_{l-1}}\left[\widehat{\boldsymbol{s}}_{l}, \boldsymbol{Y}_{j}\right]=\frac{1}{\beta_{l-1}}\left[\left(\Lambda-\alpha_{l} I_{n}\right) \boldsymbol{Y}_{l}-\gamma_{l} \boldsymbol{Y}_{l+1}, \boldsymbol{Y}_{j}\right] \\
& =\frac{1}{\beta_{l-1}}\left(\left[\Lambda \boldsymbol{Y}_{l}, \boldsymbol{Y}_{j}\right]-\alpha_{l} \delta_{l j} \delta_{l}-\gamma_{l} \delta_{l+1, j} \delta_{l+1}\right) .
\end{aligned}
$$

Clearly steps 7 and 9 imply that the right-hand side of the above equality is zero for $j=l$. If $j>l$, we get

$$
\begin{aligned}
{\left[\Lambda \boldsymbol{Y}_{l}, \boldsymbol{Y}_{j}\right] } & =\left[\boldsymbol{Y}_{l}, \Lambda \boldsymbol{Y}_{j}\right]=\left[\boldsymbol{Y}_{l}, \widehat{\boldsymbol{s}}_{j}+\alpha_{j} \boldsymbol{Y}_{j}+\gamma_{j} \boldsymbol{Y}_{j+1}\right] \\
& =\beta_{j-1} \delta_{l, j-1} \delta_{l}+\alpha_{j} \delta_{l, j} \delta_{l}+\gamma_{j} \delta_{l, j+1} \delta_{l}=\beta_{j-1} \delta_{l, j-1} \delta_{l}
\end{aligned}
$$

from steps 6 and 10. Then

$$
\left[\boldsymbol{Y}_{l-1}, \boldsymbol{Y}_{j}\right]=\frac{1}{\beta_{l-1}}\left(\beta_{j-1} \delta_{l, j-1} \delta_{l}-\gamma_{l} \delta_{l+1, j} \delta_{l+1}\right)=0,
$$

because $\gamma_{k-1}=\frac{1}{\beta_{k-1}}\left[\widehat{\boldsymbol{s}}_{k}, \widehat{\boldsymbol{s}}_{k}\right] \delta_{k}=\epsilon_{k-1} \beta_{k-1}$, for $k=n, n-1, \ldots, 2$, from steps 4,5 , and 11.

Next, we show that the matrix $Z=\left[\boldsymbol{Z}_{1}, \boldsymbol{Z}_{2}, \ldots, \boldsymbol{Z}_{n}\right]$ computed by this algorithm is an $H$-orthogonal matrix. As

$$
\left[\boldsymbol{Z}_{i}, \boldsymbol{Z}_{j}\right]=\left[\boldsymbol{Y}_{i}, \boldsymbol{Y}_{j}\right] \delta_{i} \delta_{j}=\delta_{i j} \delta_{j}
$$

for $j=n, n-1, \ldots, i$, and $i=n, n-1, \ldots, 1$, from step 7 and (A.3), we can also get $Z=H Y H$ as well as the biorthogonality condition $Y^{\mathrm{T}} Z=I_{n}$.

Now, we show that $\widehat{\boldsymbol{s}}_{1}=\mathbf{0}$. It is sufficient to prove that $\left[\widehat{\boldsymbol{s}}_{1}, \boldsymbol{Y}_{i}\right]=0$, for $i=1,2, \ldots, n$, because $\boldsymbol{Y}_{1}, \boldsymbol{Y}_{2}, \ldots, \boldsymbol{Y}_{n}$ constitute an $H$-orthonormal basis of $\mathbb{R}^{n}$. If $i=1$, we obtain

$$
\left[\widehat{\boldsymbol{s}}_{1}, \boldsymbol{Y}_{1}\right]=\left[\Lambda \boldsymbol{Y}_{1}-\alpha_{1} \boldsymbol{Y}_{1}-\gamma_{1} \boldsymbol{Y}_{2}, \boldsymbol{Y}_{1}\right]=\left[\Lambda \boldsymbol{Y}_{1}, \boldsymbol{Y}_{1}\right]-\alpha_{1} \delta_{1}=0
$$

from steps 7, 9, and 10. If $i=2$, we have

$$
\begin{aligned}
{\left[\widehat{\boldsymbol{s}}_{1}, \boldsymbol{Y}_{2}\right] } & =\left[\Lambda \boldsymbol{Y}_{1}-\alpha_{1} \boldsymbol{Y}_{1}-\gamma_{1} \boldsymbol{Y}_{2}, \boldsymbol{Y}_{2}\right]=\left[\boldsymbol{Y}_{1}, \Lambda \boldsymbol{Y}_{2}\right]-\gamma_{1} \delta_{2} \\
& =\left[\boldsymbol{Y}_{1}, \widehat{\boldsymbol{s}}_{2}+\alpha_{2} \boldsymbol{Y}_{2}+\gamma_{2} \boldsymbol{Y}_{3}\right]-\gamma_{1} \delta_{2}=\beta_{1} \delta_{1}-\gamma_{1} \delta_{2}=0
\end{aligned}
$$

from steps 6 and 10 , and $\gamma_{1}=\epsilon_{1} \beta_{1}$. If $i \geq 3$, we obtain

$$
\begin{aligned}
{\left[\widehat{\boldsymbol{s}}_{1}, \boldsymbol{Y}_{i}\right] } & =\left[\Lambda \boldsymbol{Y}_{1}-\alpha_{1} \boldsymbol{Y}_{1}-\gamma_{1} \boldsymbol{Y}_{2}, \boldsymbol{Y}_{i}\right]=\left[\boldsymbol{Y}_{1}, \Lambda \boldsymbol{Y}_{i}\right] \\
& =\left[\boldsymbol{Y}_{1}, \widehat{\boldsymbol{s}}_{i}+\alpha_{i} \boldsymbol{Y}_{i}+\gamma_{i} \boldsymbol{Y}_{i+1}\right]=\left[\boldsymbol{Y}_{1}, \beta_{i-1} \boldsymbol{Y}_{i-1}\right]=0
\end{aligned}
$$

from steps 6 and 10. Then, $\widehat{\boldsymbol{s}}_{1}=\widehat{\boldsymbol{r}}_{1}=\mathbf{0}$ holds from step 11. Thus, the algorithm will prematurely terminate in this case and it follows that

$$
\Lambda Y=Y \widehat{J}^{\mathrm{T}} \quad \text { and } \quad \Lambda Z=Z \widehat{J}
$$

and so $\left(Y^{-1}\right)^{\mathrm{T}} \widehat{J} Y^{\mathrm{T}}=\Lambda$.

Finally, we demonstrate that the constructed pseudo-Jacobi matrix $\widehat{J}$ is unique. Let $\widetilde{J}$ be a pseudo-Jacobi matrix characterized by the distinct real eigenvalues $\widehat{\lambda}_{1}, \widehat{\lambda}_{2}, \ldots, \widehat{\lambda}_{n}$, the nonzero $H$-orthonormal vector $\left(\boldsymbol{v}_{n, 1}, \boldsymbol{v}_{n, 2}, \ldots, \boldsymbol{v}_{n, n}\right)^{\mathrm{T}}$, and the pseudo-norms $\delta_{1}, \delta_{2}, \ldots, \delta_{n}$. The column vectors $\widetilde{\boldsymbol{Y}}_{n}, \widetilde{\boldsymbol{Y}}_{n-1}, \ldots, \widetilde{\boldsymbol{Y}}_{1}$, obtained by the algorithm, are pseudo-orthogonal with the 
respective norms $\delta_{n}, \delta_{n-1}, \ldots, \delta_{1}$. The computed column vectors $\widetilde{\boldsymbol{Z}}_{n}, \widetilde{\boldsymbol{Z}}_{n-1}, \ldots, \widetilde{\boldsymbol{Z}}_{1}$ are also pseudo-orthogonal and satisfy the biorthogonality condition $\widetilde{\boldsymbol{Y}}_{i}^{\mathrm{T}} \widetilde{\boldsymbol{Z}}_{j}=\delta_{i j}$, for $i, j=1,2, \ldots, n$. Thus,

$$
\Lambda \widetilde{Y}=\widetilde{Y} \widetilde{J}^{\mathrm{T}} \quad \text { and } \quad \Lambda \widetilde{Z}=\widetilde{Z} \widetilde{J}
$$

for $\widetilde{Y}=\left[\widetilde{\boldsymbol{Y}}_{1}, \widetilde{\boldsymbol{Y}}_{2}, \ldots, \widetilde{\boldsymbol{Y}}_{n}\right]$ and $\widetilde{Z}=\left[\widetilde{\boldsymbol{Z}}_{1}, \widetilde{\boldsymbol{Z}}_{2}, \ldots, \widetilde{\boldsymbol{Z}}_{n}\right]$. Because all the subdiagonal entries $\widetilde{\beta}_{i}$ and the superdiagonal entries $\widetilde{\gamma}_{i}$ of $\widetilde{J}$ satisfy $\widetilde{\gamma}_{i}=\epsilon_{i} \widetilde{\beta}_{i}$, we only need to prove that all the main diagonal entries $\widetilde{\alpha}_{i}$ and subdiagonal entries $\widetilde{\beta}_{i}$ of $\widetilde{J}$ are equal to the corresponding entries $\alpha_{i}$ and $\beta_{i}$ of the matrix $\widehat{J}$ computed by the algorithm.

From the identities in (A.4), the columns $\widetilde{\boldsymbol{Y}}_{k}$ and $\widetilde{\boldsymbol{Z}}_{k}$ of the $H$-orthogonal matrices $Y$ and $Z$, respectively, satisfy the following recurrence relations:

$$
\begin{aligned}
\widetilde{\beta}_{k-1} \widetilde{\boldsymbol{Y}}_{k-1} & =\widetilde{\boldsymbol{s}}_{k}=\left(\Lambda-\widetilde{\alpha}_{k} I_{n}\right) \widetilde{\boldsymbol{Y}}_{k}-\widetilde{\gamma}_{k} \widetilde{\boldsymbol{Y}}_{k+1}, \\
\widetilde{\gamma}_{k-1} \widetilde{\boldsymbol{Z}}_{k-1} & =\widetilde{\boldsymbol{r}}_{k}=\left(\Lambda-\widetilde{\alpha}_{k} I_{n}\right) \widetilde{\boldsymbol{Z}}_{k}-\widetilde{\beta}_{k} \widetilde{\boldsymbol{Z}}_{k+1}
\end{aligned}
$$

for $k=n, n-1, \ldots, 1$, with $\widetilde{\boldsymbol{Y}}_{n+1}=\widetilde{\boldsymbol{Z}}_{n+1}=\mathbf{0}$ and $\widetilde{\boldsymbol{s}}_{1}=\widetilde{\boldsymbol{r}}_{1}=\mathbf{0}$. As $\widetilde{\boldsymbol{Z}}_{k}=H \widetilde{\boldsymbol{Y}}_{k} \delta_{k}$ and $\widetilde{\boldsymbol{r}}_{k}=H \widetilde{\boldsymbol{s}}_{k} \delta_{k}$, we only consider the first recurrence relation pre-multiplied by $\widetilde{\boldsymbol{Z}}_{k}^{\mathrm{T}}$. It follows that

$$
\widetilde{\alpha}_{k}=\widetilde{\boldsymbol{Z}}_{k}^{\mathrm{T}} \Lambda \widetilde{\boldsymbol{Y}}_{k}, \quad k=n, n-1, \ldots, 1
$$

Observing that

$$
1=\widetilde{\boldsymbol{Y}}_{k-1}^{\mathrm{T}} \widetilde{\boldsymbol{Z}}_{k-1}=\frac{1}{\widetilde{\beta}_{k-1} \widetilde{\gamma}_{k-1}} \widetilde{\boldsymbol{s}}_{k}^{\mathrm{T}} \widetilde{\boldsymbol{r}}_{k}
$$

we find that

$$
\widetilde{\boldsymbol{Y}}_{k-1}=\frac{1}{\widetilde{\beta}_{k-1}} \widetilde{\boldsymbol{s}}_{k}, \quad \widetilde{\gamma}_{k-1}=\frac{1}{\widetilde{\beta}_{k-1}} \widetilde{\boldsymbol{s}}_{k}^{\mathrm{T}} \widetilde{\boldsymbol{r}}_{k},
$$

and so

$$
\left[\widetilde{\beta}_{k-1} \widetilde{\boldsymbol{Y}}_{k-1}, \widetilde{\beta}_{k-1} \widetilde{\boldsymbol{Y}}_{k-1}\right]=\left[\widetilde{\boldsymbol{s}}_{k}, \widetilde{\boldsymbol{s}}_{k}\right]
$$

Thus,

$$
\widetilde{\beta}_{k-1}=\sqrt{\left[\widetilde{\boldsymbol{s}}_{k}, \widetilde{\boldsymbol{s}}_{k}\right] \delta_{k-1}}, \quad k=n, n-1, \ldots, 2 .
$$

By the sequence of computations in the backward modified unsymmetric Lanczos algorithm and considering $\widetilde{\boldsymbol{Y}}_{n}=\left(\boldsymbol{v}_{n, 1}, \boldsymbol{v}_{n, 2}, \ldots, \boldsymbol{v}_{n, n}\right)^{\mathrm{T}}$, we can easily show that the entries $\widetilde{\alpha}_{n}, \widetilde{\beta}_{n-1}$, $\widetilde{\alpha}_{n-1}, \widetilde{\beta}_{n-2}, \ldots, \widetilde{\beta}_{1}, \widetilde{\alpha}_{1}$ of $\widetilde{J}$ coincide, respectively, with the entries $\alpha_{n}, \beta_{n-1}, \alpha_{n-1}, \beta_{n-2}$, $\ldots, \beta_{1}, \alpha_{1}$ of $\widehat{J}$.

REMARK A.5. By executing the algorithm in Theorem 2.3, we can also get $Y=V^{\mathrm{T}}$ and $Z=V^{\#}$ from the initial vector $\boldsymbol{Y}_{n}=\left(\boldsymbol{v}_{n, 1}, \boldsymbol{v}_{n, 2}, \ldots, \boldsymbol{v}_{n, n}\right)^{\mathrm{T}}$.

Appendix B. Proofs of Theorems 3.1 and 3.2. The proofs of the theorems in Section 3 use the following four lemmas. The first one gives a necessary and sufficient condition for $J_{r}$ and $J_{r+2, n}$ in (3.1) to share a common eigenvalue. As noticed by one of the referees, the 
rational function $F_{1}(\lambda)$ there appearing is the $(r+1, r+1)$-entry of the twisted factorization of the tridiagonal matrix $\lambda I_{n}-J_{n}$ (see [22]).

LemmA B.1. Let $\boldsymbol{\mu}_{1}$ and $\boldsymbol{\mu}_{2}$ be the spectra of $J_{r}$ and $J_{r+2, n}$ in (3.1), respectively. Then, an element of $\boldsymbol{\mu}_{1}$ is an eigenvalue of $J_{n}$ in (3.1) if and only if it is an element of $\boldsymbol{\mu}_{2}$ and vice-versa.

Proof. From (3.2), it follows that $U=U_{1} \oplus I_{1} \oplus U_{2}$ is a pseudo-orthogonal matrix with respect to $\widetilde{H}=H_{1} \oplus I_{1} \oplus H_{2}$, and so $U^{\#}=\widetilde{H} U^{\mathrm{T}} \widetilde{H}$. Thus, we get

$$
U^{\#} J_{n} U=\left[\begin{array}{ccc}
\Lambda_{1} & \epsilon_{r} \beta_{r} U_{1}^{\#} \mathbf{e}_{r} & \mathbf{0} \\
\beta_{r} \mathbf{e}_{r}^{\mathrm{T}} U_{1} & \alpha_{r+1} & \epsilon_{r+1} \beta_{r+1} \boldsymbol{\omega}_{1}^{\mathrm{T}} U_{2} \\
\mathbf{0} & \beta_{r+1} U_{2}^{\#} \boldsymbol{\omega}_{1} & \Lambda_{2}
\end{array}\right] .
$$

By using the Laplace expansion for the determinant, it follows that

(B.1)

$$
\begin{aligned}
& \operatorname{det}\left(\lambda I_{n}-J_{n}\right)=\operatorname{det}\left(\lambda I_{n}-U^{\#} J_{n} U\right) \\
& =\prod_{j=1}^{n-1}\left(\lambda-\mu_{j}\right)\left(\lambda-\alpha_{r+1}-\sum_{i=1}^{r} \frac{\delta_{r+1}\left(\beta_{r} u_{r, i}^{(1)}\right)^{2} \delta_{i}}{\lambda-\mu_{i}}-\sum_{i=r+1}^{n-1} \frac{\delta_{r+1}\left(\beta_{r+1} u_{1, i-r}^{(2)}\right)^{2} \delta_{i+1}}{\lambda-\mu_{i}}\right) .
\end{aligned}
$$

Because $\beta_{r} u_{r, i}^{(1)} \neq 0, i=1,2, \ldots, r$, and $\beta_{r+1} u_{1, i-r}^{(2)} \neq 0, i=r+1, r+2, \ldots, n-1$, from Lemma A.4 (1) we find that

$$
\begin{aligned}
& \operatorname{det}\left(\mu_{j} I_{n}-J_{n}\right) \\
& \quad=-\prod_{i=1, i \neq j}^{n-1}\left(\mu_{j}-\mu_{i}\right) \cdot \begin{cases}\delta_{r+1}\left(\beta_{r} u_{r, j}^{(1)}\right)^{2} \delta_{j}, & j=1,2, \ldots, r \\
\delta_{r+1}\left(\beta_{r+1} u_{1, j-r}^{(2)}\right)^{2} \delta_{j+1}, & j=r+1, r+2, \ldots, n-1 .\end{cases}
\end{aligned}
$$

Due to the fact that all the elements in $\boldsymbol{\mu}_{1}$ and $\boldsymbol{\mu}_{2}$ are pairwise distinct, $\mu_{j}$ is an eigenvalue of $J_{n}$ if and only if $\prod_{i=1, i \neq j}^{n-1}\left(\mu_{j}-\mu_{i}\right)=0$, that is, $\mu_{j}$ is a common eigenvalue of $J_{r}$ and $J_{r+2, n}$.

If $J_{r}$ and $J_{r+2, n}$ have no common eigenvalues, then the following holds.

Lemma B.2. Let $\sigma\left(J_{r}\right)=\boldsymbol{\mu}_{1}$ and $\sigma\left(J_{r+2, n}\right)=\boldsymbol{\mu}_{2}$. If $\sigma\left(J_{r}\right) \cap \sigma\left(J_{r+2, n}\right)=\varnothing$, then the eigenvalues of $J_{n}$ are the $n$ zeros of the following rational function

$$
F_{1}(\lambda)=\lambda-\alpha_{r+1}-\sum_{i=1}^{r} \frac{\delta_{r+1}\left(\beta_{r} u_{r, i}^{(1)}\right)^{2} \delta_{i}}{\lambda-\mu_{i}}-\sum_{i=r+1}^{n-1} \frac{\delta_{r+1}\left(\beta_{r+1} u_{1, i-r}^{(2)}\right)^{2} \delta_{i+1}}{\lambda-\mu_{i}} .
$$

Proof. By Lemma B.1, $\mu_{j} \notin \sigma\left(J_{n}\right)$, for any $j=1,2, \ldots, n-1$, if $\sigma\left(J_{r}\right) \cap \sigma\left(J_{r+2, n}\right)=\varnothing$. Then, $\operatorname{det}\left(\lambda I_{n}-J_{n}\right)=0$ is equivalent to

$$
F_{1}(\lambda)=\lambda-\alpha_{r+1}-\sum_{i=1}^{r} \frac{\delta_{r+1}\left(\beta_{r} u_{r, i}^{(1)}\right)^{2} \delta_{i}}{\lambda-\mu_{i}}-\sum_{i=r+1}^{n-1} \frac{\delta_{r+1}\left(\beta_{r+1} u_{1, i-r}^{(2)}\right)^{2} \delta_{i+1}}{\lambda-\mu_{i}}=0
$$

from equation (B.1), and the result holds.

If $J_{r}$ and $J_{r+2, n}$ have common eigenvalues, then the following holds.

Lemma B.3. Let $\sigma\left(J_{r}\right)=\boldsymbol{\mu}_{1}$ and $\sigma\left(J_{r+2, n}\right)=\boldsymbol{\mu}_{2}$. Assume $\boldsymbol{\mu}_{1} \cap \boldsymbol{\mu}_{2}=\left\{\mu_{i}\right\}_{i=1}^{k}$ and $\mu_{r+i}=\mu_{i}$, for any $i=1,2, \ldots, k$, with $k \leq \min \{r, n-r-1\}$. Then, $\mu_{1}, \mu_{2}, \ldots, \mu_{k}$ are 
eigenvalues of $J_{n}$, and the remaining eigenvalues of $J_{n}$ are the $n-k$ zeros of the rational function

$$
\begin{aligned}
F_{2}(\lambda)=\lambda & -\alpha_{r+1}-\sum_{i=1}^{k} \frac{\delta_{r+1}\left(\beta_{r} u_{r, i}^{(1)}\right)^{2} \delta_{i}+\delta_{r+1}\left(\beta_{r+1} u_{1, i}^{(2)}\right)^{2} \delta_{r+i+1}}{\lambda-\mu_{i}} \\
& -\sum_{i=k+1}^{r} \frac{\delta_{r+1}\left(\beta_{r} u_{r, i}^{(1)}\right)^{2} \delta_{i}}{\lambda-\mu_{i}}-\sum_{i=r+k+1}^{n-1} \frac{\delta_{r+1}\left(\beta_{r+1} u_{1, i-r}^{(2)}\right)^{2} \delta_{i+1}}{\lambda-\mu_{i}} .
\end{aligned}
$$

Proof. Because $\mu_{i} \in \sigma\left(J_{r}\right) \cap \sigma\left(J_{r+2, n}\right)$, for any $i=1,2, \ldots, k$, then $\mu_{1}, \mu_{2}, \ldots, \mu_{k}$ are also eigenvalues of $J_{n}$ by Lemma B.1. Hence the remaining eigenvalues of $J_{n}$ are the zeros of the polynomial

$$
G(\lambda)=\frac{\operatorname{det}\left(\lambda I_{n}-J_{n}\right)}{\prod_{i=1}^{k}\left(\lambda-\mu_{i}\right)}=\prod_{j=k+1}^{n-1}\left(\lambda-\mu_{j}\right) F_{1}(\lambda)
$$

from equation (B.1). Since $\mu_{i} \notin \sigma\left(J_{r}\right) \cap \sigma\left(J_{r+2, n}\right)$ for any $i \notin\{1,2, \ldots, k\} \cup\{r+1, r+$ $2, \ldots, r+k\}$, from Lemma B. $1 \prod_{j=k+1}^{n-1}\left(\lambda-\mu_{j}\right) \neq 0$ for any $\lambda \notin\left\{\mu_{1}, \mu_{2}, \ldots, \mu_{k}\right\}$. Hence, $G(\lambda)=0$ if and only if

$$
\begin{aligned}
F_{2}(\lambda)=F_{1}(\lambda)=\lambda & -\alpha_{r+1}-\sum_{i=1}^{k} \frac{\delta_{r+1}\left(\beta_{r} u_{r, i}^{(1)}\right)^{2} \delta_{i}+\delta_{r+1}\left(\beta_{r+1} u_{1, i}^{(2)}\right)^{2} \delta_{r+i+1}}{\lambda-\mu_{i}} \\
& -\sum_{i=k+1}^{r} \frac{\delta_{r+1}\left(\beta_{r} u_{r, i}^{(1)}\right)^{2} \delta_{i}}{\lambda-\mu_{i}}-\sum_{i=r+k+1}^{n-1} \frac{\delta_{r+1}\left(\beta_{r+1} u_{1, i-r}^{(2)}\right)^{2} \delta_{i+1}}{\lambda-\mu_{i}}=0 .
\end{aligned}
$$

By construction, $G(\lambda)$ has degree $n-k$ and so $G(\lambda)$ has $n-k$ zeros. Thus, $F_{2}(\lambda)$ also has $n-k$ zeros.

In order to prove the main theorems in Section 3, we recall the following crucial result presented in $[5,17]$.

LEMMA B.4. Let $\left\{\xi_{1}, \xi_{2}, \ldots, \xi_{m}\right\}$ be a set of complex numbers closed under conjugation, and let $\left\{\eta_{1}, \eta_{2}, \ldots, \eta_{m-1}\right\}$ be a set of distinct real numbers with $\eta_{j} \notin\left\{\xi_{1}, \xi_{2} \ldots \xi_{m}\right\}$. Then, the following system of linear algebraic equations

$$
\frac{x_{1}}{\xi_{i}-\eta_{1}}+\frac{x_{2}}{\xi_{i}-\eta_{2}}+\cdots+\frac{x_{m-1}}{\xi_{i}-\eta_{m-1}}=\xi_{i}-a, \quad i=1,2, \ldots, m
$$

has a unique solution $\boldsymbol{x}=\left(x_{1}, x_{2}, \ldots, x_{m-1}\right)$ if and only if

$$
x_{j}=-\frac{\prod_{i=1}^{m}\left(\xi_{i}-\eta_{j}\right)}{\prod_{i=1, i \neq j}^{m-1}\left(\eta_{i}-\eta_{j}\right)}, \quad j=1,2, \ldots, m-1,
$$

and $a=\sum_{i=1}^{m} \xi_{i}-\sum_{i=1}^{m-1} \eta_{i}$.

Proof of Theorem 3.1. Necessity: Assume that there exists a pseudo-Jacobi matrix $J_{n} \in \mathcal{J}(n, \boldsymbol{\epsilon}, \boldsymbol{\beta})$ as in (3.1) such that $\sigma\left(J_{n}\right)=\boldsymbol{\lambda}, \sigma\left(J_{r}\right)=\boldsymbol{\mu}_{1}$, and $\sigma\left(J_{r+2, n}\right)=\boldsymbol{\mu}_{2}$. Because $\boldsymbol{\mu}_{1} \cap \boldsymbol{\mu}_{2}=\varnothing$, then it follows from Lemma B.2 that the eigenvalues of $J_{n}$ are the zeros of $F_{1}(\lambda)=0$ in (B.2). By Lemma B.4, we get

$$
\begin{cases}\delta_{r+1}\left(\beta_{r} u_{r, j}^{(1)}\right)^{2} \delta_{j}=x_{j}, & j=1,2, \ldots, r, \\ \delta_{r+1}\left(\beta_{r+1} u_{1, j-r}^{(2)}\right)^{2} \delta_{j+1}=x_{j}, & j=r+1, r+2, \ldots, n-1,\end{cases}
$$


where $x_{j}=-\prod_{i=1}^{n}\left(\lambda_{i}-\mu_{j}\right) \prod_{i=1, i \neq j}^{n-1}\left(\mu_{i}-\mu_{j}\right)^{-1}$. Since $\beta_{r}, \beta_{r+1}, u_{r, j}^{(1)}, j=1,2, \ldots, r$, and $u_{1, j-r}^{(2)}, j=r+1, r+2, \ldots, n-1$, are real, then condition (1) holds.

By (3.2) we know that $\sum_{j=1}^{r}\left(u_{r, j}^{(1)}\right)^{2} \delta_{j}=\delta_{r}$ and $\sum_{j=r+1}^{n-1}\left(u_{1, j-r}^{(2)}\right)^{2} \delta_{j+1}=\delta_{r+2}$. Thus, condition (2) follows from (B.4). Finally, conditions (3) and (4) are satisfied because

$$
\begin{aligned}
\beta_{k-1}^{2} & =\left[\widehat{\boldsymbol{s}}_{k}, \widehat{\boldsymbol{s}}_{k}\right] \delta_{k-1}>0, & & \text { for } k=r, r-1, \ldots, 2, \text { and } \\
\beta_{r+k+1}^{2} & =\left[\boldsymbol{r}_{r+k+1}, \boldsymbol{r}_{r+k+1}\right] \delta_{r+k+2} / \delta_{r+2}>0, & & \text { for } k=1,2, \ldots, n-r-2 .
\end{aligned}
$$

Sufficiency: Assume that conditions (1)-(4) hold. Consider the sign vector $\epsilon$ and the nonzero real numbers

$$
x_{j}=-\frac{\prod_{i=1}^{n}\left(\lambda_{i}-\mu_{j}\right)}{\prod_{i=1, i \neq j}^{n-1}\left(\mu_{i}-\mu_{j}\right)}, \quad j=1,2, \ldots, n-1 .
$$

Let us define

$$
\beta_{r}:=\left(\epsilon_{r} \sum_{j=1}^{r} x_{j}\right)^{\frac{1}{2}}, \quad \beta_{r+1}:=\left(\epsilon_{r+1} \sum_{j=r+1}^{n-1} x_{j}\right)^{\frac{1}{2}}
$$

and

$$
\begin{cases}u_{r, j}^{(1)}:=\frac{\sqrt{\delta_{r+1} \delta_{j} x_{j}}}{\beta_{r}}, & j=1,2, \ldots, r, \\ u_{1, j-r}^{(2)}:=\frac{\sqrt{\delta_{r+1} \delta_{j+1} x_{j}}}{\beta_{r+1}}, & j=r+1, r+2, \ldots, n-1 .\end{cases}
$$

Then, $g_{1}=\left(u_{r, 1}^{(1)}, u_{r, 2}^{(1)}, \ldots, u_{r, r}^{(1)}\right)^{\mathrm{T}}$ and $g_{2}=\left(u_{11}^{(2)}, u_{12}^{(2)}, \ldots, u_{1, n-r-1}^{(2)}\right)^{\mathrm{T}}$ are, respectively, an $H_{1}$-orthonormal and an $H_{2}$-orthonormal vector. Furthermore, condition (3) ensures that a unique pseudo-Jacobi matrix $J_{r}$ can be constructed from $\left(H_{1}, \boldsymbol{\mu}_{1}, g_{1}\right)$ by using the algorithm in Theorem 2.2. Similarly, condition (4) guarantees that the algorithm in Theorem 2.3 generates a unique pseudo-Jacobi matrix $J_{r+2, n}$ given $\left(H_{2}, \boldsymbol{\mu}_{2}, g_{2}\right)$. Since $\alpha_{r+1}=\sum_{i=1}^{n} \lambda_{i}-\sum_{i=1}^{n-1} \mu_{i}$, a unique pseudo-Jacobi matrix $J_{n}$ is so constructed.

From equations (B.5) and (B.7), we find

$$
x_{j}= \begin{cases}\delta_{r+1}\left(\beta_{r} u_{r, j}^{(1)}\right)^{2} \delta_{j}, & j=1,2, \ldots, r, \\ \delta_{r+1}\left(\beta_{r+1} u_{1, j-r}^{(2)}\right)^{2} \delta_{j+1}, & j=r+1, r+2, \ldots, n-1,\end{cases}
$$

By Lemma B.4, $F_{1}\left(\lambda_{i}\right)=0$ holds for $i=1,2, \ldots, n$, in Lemma B.2. Thus, it follows that $\operatorname{det}\left(\lambda_{i} I_{n}-J_{n}\right)=0, i=1,2, \ldots, n$. Therefore, $\boldsymbol{\lambda}=\sigma\left(J_{n}\right)$ and the constructed matrix $J_{n}$ is the unique solution of the PJIEP.

Proof of Theorem 3.2. Necessity: For the given sign vector $\epsilon$ and the sets $\lambda, \boldsymbol{\mu}_{1}$, and $\boldsymbol{\mu}_{2}$, suppose that there exists a pseudo-Jacobi matrix $J_{n} \in \mathcal{J}(n, \boldsymbol{\epsilon}, \boldsymbol{\beta})$ as in (3.1). If $\boldsymbol{\mu}_{1} \cap \boldsymbol{\mu}_{2}=\left\{\mu_{i}\right\}_{i=1}^{k}$ and $\mu_{r+i}=\mu_{i}$, for $i=1,2, \ldots, k$, then $\lambda_{i}=\mu_{i}, i=1,2, \ldots, k$, are also the eigenvalues of $J_{n}$ by Lemma B.1. The remaining eigenvalues $\lambda_{k+1}, \lambda_{k+2}, \ldots, \lambda_{n}$ of $J_{n}$ are the zeros of $F_{2}(\lambda)=0$ in (B.3) by Lemma B.3. Thus, from Lemma B.4 we obtain

$$
\begin{aligned}
\delta_{r+1}\left(\beta_{r} u_{r, j}^{(1)}\right)^{2} \delta_{j}+\delta_{r+1}\left(\beta_{r+1} u_{1, j}^{(2)}\right)^{2} \delta_{r+j+1} & =x_{r+j}, & & j=1,2, \ldots, k, \\
\delta_{r+1}\left(\beta_{r} u_{r, j}^{(1)}\right)^{2} \delta_{j} & =x_{j}, & & j=k+1, k+2, \ldots, r, \\
\delta_{r+1}\left(\beta_{r+1} u_{1, j-r}^{(2)}\right)^{2} \delta_{j+1} & =x_{j}, & & j=r+k+1, \ldots, n-1,
\end{aligned}
$$


where $x_{j}=-\prod_{i=k+1}^{n}\left(\lambda_{i}-\mu_{j}\right) \prod_{i=k+1, i \neq j}^{n-1}\left(\mu_{i}-\mu_{j}\right)^{-1}, j=k+1, \ldots, n-1$. Then there exist real numbers $\theta_{j} \notin\{0,1\}$ such that

$$
\delta_{r+1}\left(\beta_{r} u_{r, j}^{(1)}\right)^{2} \delta_{j}=\theta_{j} x_{r+j}, \quad \delta_{r+1}\left(\beta_{r+1} u_{1, j}^{(2)}\right)^{2} \delta_{r+j+1}=\left(1-\theta_{j}\right) x_{r+j},
$$

for $j=1,2, \ldots, k$. Having in mind that $\beta_{r} u_{r, j}^{(1)} \neq 0, j=1,2, \ldots, r$, and $\beta_{r+1} u_{1, j}^{(2)} \neq 0$, $j=1,2, \ldots, n-r-1$, from Lemma A.4, conditions (1) and (2) are satisfied.

Because $\sum_{j=1}^{r}\left(u_{r, j}^{(1)}\right)^{2} \delta_{j}=\delta_{r}$ and $\sum_{j=r+1}^{n-1}\left(u_{1, j-r}^{(2)}\right)^{2} \delta_{j+1}=\delta_{r+2}$ from (3.2), condition (3) holds by (B.8) and (B.9). Since $\beta_{k-1}^{2}=\left[\widehat{\boldsymbol{s}}_{k}, \widehat{\boldsymbol{s}}_{k}\right] \delta_{k-1}>0$ for $k=r, r-1, \ldots, 2$, and $\beta_{r+k+1}^{2}=\left[\boldsymbol{r}_{r+k+1}, \boldsymbol{r}_{r+k+1}\right] \delta_{r+k+2} / \delta_{r+2}>0$ for $k=1,2, \ldots, n-r-2$, conditions (3) and (4) in Theorem 3.1 follow.

Sufficiency: Because $\boldsymbol{\mu}_{1} \cap \boldsymbol{\mu}_{2}=\left\{\mu_{i}\right\}_{i=1}^{k}$, then $\lambda_{i}=\mu_{i}, i \in\{1,2, \ldots, k\}$ are eigenvalues of a pseudo-Jacobi matrix $J_{n}$ which will be constructed in the sequel. If the conditions in this theorem are satisfied, let

$$
x_{j}=-\frac{\prod_{i=k+1}^{n}\left(\lambda_{i}-\mu_{j}\right)}{\prod_{i=k+1, i \neq j}^{n-1}\left(\mu_{i}-\mu_{j}\right)}, \quad j=k+1, \ldots, n-1,
$$

where $x_{j}$ are all nonzero real numbers. For the selected $\theta_{j} \in \mathbb{R}-\{0,1\}, j=1,2, \ldots, k$, let us define

$$
\beta_{r}:=\left(\epsilon_{r}\left(\sum_{j=1}^{k} \theta_{j} x_{r+j}+\sum_{j=k+1}^{r} x_{j}\right)\right)^{\frac{1}{2}}
$$

$$
\beta_{r+1}:=\left(\epsilon_{r+1}\left(\sum_{j=1}^{k}\left(1-\theta_{j}\right) x_{r+j}+\sum_{j=r+k+1}^{n-1} x_{j}\right)\right)^{\frac{1}{2}},
$$

$$
u_{r, j}^{(1)}:= \begin{cases}\frac{1}{\beta_{r}} \sqrt{\delta_{r+1} \delta_{j} \theta_{j} x_{r+j}}, & j=1,2, \ldots, k \\ \frac{1}{\beta_{r}} \sqrt{\delta_{r+1} \delta_{j} x_{j}}, & j=k+1, \ldots, r\end{cases}
$$

and

$$
u_{1, j}^{(2)}:= \begin{cases}\frac{1}{\beta_{r+1}} \sqrt{\delta_{r+1} \delta_{r+j+1}\left(1-\theta_{j}\right) x_{r+j}}, & j=1,2, \ldots, k, \\ \frac{1}{\beta_{r+1}} \sqrt{\delta_{r+1} \delta_{r+j+1} x_{r+j}}, & j=k+1, \ldots, n-r-1 .\end{cases}
$$

Hence, we can construct a unique Jacobi matrix $J_{r}$ from $H_{1}, \boldsymbol{\mu}_{1}$ and also the $H_{1}$-orthonormal vector $g_{1}=\left(u_{r, 1}^{(1)}, u_{r, 2}^{(1)}, \ldots, u_{r, r}^{(1)}\right)^{\mathrm{T}}$ by using the algorithm in Theorem 2.2 with the help of condition (3) in Theorem 3.1. In addition, condition (4) in Theorem 3.1 ensures that a unique pseudo-Jacobi matrix $J_{r+2, n}$ can be constructed from $H_{2}, \boldsymbol{\mu}_{2}$ and the $H_{2}$-orthonormal vector $g_{2}=\left(u_{11}^{(2)}, u_{12}^{(2)}, \ldots, u_{1, n-r-1}^{(2)}\right)^{\mathrm{T}}$ by using the algorithm in Theorem 2.3. Then, $\alpha_{r+1}=\sum_{i=1}^{n} \lambda_{i}-\sum_{i=1}^{n-1} \mu_{i}$ from (3.1), and a pseudo-Jacobi matrix $J_{n}$ is so reconstructed.

As $\beta_{r}, \beta_{r+1}, u_{r, j}^{(1)}$, and $u_{1, j}^{(2)}$ depend on $\theta_{j} \in \mathbb{R}-\{0,1\}$ and the $\theta_{j}$ can be taken arbitrarily for $j=1,2, \ldots, k$, there are infinite pseudo-Jacobi matrices $J_{r}$ and $J_{r+2, n}$ obtained from the algorithms in Theorems 2.2 and 2.3, and so infinite pseudo-Jacobi matrices $J_{n}$ can be achieved. 
Finally, we show that a reconstructed pseudo-Jacobi matrix $J_{n}$ solves the PJIEP. By equations (B.10), (B.12), and (B.13), we have

$$
x_{j}= \begin{cases}\delta_{r+1}\left(\beta_{r} u_{r, j-r}^{(1)}\right)^{2} \delta_{j-r}+\delta_{r+1}\left(\beta_{r+1} u_{1, j-r}^{(2)}\right)^{2} \delta_{j+1}, & j=r+1, r+2, \ldots, r+k, \\ \delta_{r+1}\left(\beta_{r} u_{r, j}^{(1)}\right)^{2} \delta_{j}, & j=k+1, k+2, \ldots, r, \\ \delta_{r+1}\left(\beta_{r+1} u_{1, j-r}^{(2)}\right)^{2} \delta_{j+1}, & j=r+k+1, \ldots, n-1,\end{cases}
$$

where $\delta_{r+1}\left(\beta_{r} u_{r, j}^{(1)}\right)^{2} \delta_{j}=\theta_{j} x_{r+j}$ and $\delta_{r+1}\left(\beta_{r+1} u_{1, j}^{(2)}\right)^{2} \delta_{r+j+1}=\left(1-\theta_{j}\right) x_{r+j}, \theta_{j} \in \mathbb{R}-$ $\{0,1\}$ for $j=1,2, \ldots, k$. By Lemma B.4, $F_{2}\left(\lambda_{i}\right)=\lambda_{i}-\alpha_{r+1}-\sum_{j=k+1}^{n-1} \frac{x_{j}}{\lambda_{i}-\mu_{j}}=0$ holds for $i=k+1, k+2, \ldots, n$ in Lemma B.3. Then $\operatorname{det}\left(\lambda_{i} I_{n}-J_{n}\right)=0, i=k+1, k+2, \ldots, n$, and $\lambda_{i}, i=k+1, k+2, \ldots, n$ are the remaining eigenvalues of $J_{n}$. Thus, $\boldsymbol{\lambda}=\sigma\left(J_{n}\right)$, and $J_{n}$ is a solution of the PJIEP.

\section{REFERENCES}

[1] N. I. AkHIEZer, The Classical Moment Problem and Some Related Questions in Analysis, Hafner, New York, 1965.

[2] Y. BAI AND G. WEI, A new inverse three spectra theorem for Jacobi matrices, Linear Algebra Appl., 493 (2016), pp. 301-312.

[3] N. BEBIAno, C. M. DA Fonseca, And J. DA PRovidÊnCIA, An inverse eigenvalue problem for periodic Jacobi matrices in Minkowski spaces, Linear Algebra Appl., 435 (2011), pp. 2033-2045.

[4] N. BEBIANO AND J. DA PROVIDÊNCIA, Inverse problems for pseudo-Jacobi matrices: existence and uniqueness results, Inverse Problems, 27 (2011), Art. 025005 (12 pages).

[5] - Inverse spectral problems for structured pseudo-symmetric matrices, Linear Algebra Appl., 438 (2013), pp. 4062-4074.

[6] N. BEBIAno, S. Furtado, AND J. DA PRovidÊnCIA, An algorithm for constructing a pseudo-Jacobi matrix from given spectral data, Numer. Linear Algebra Appl., 20 (2013), pp. 185-197.

[7] D. Boley AND G.H. Golub, A survey of matrix inverse eigenvalue problem, Inverse Problems, 3 (1987), pp. 595-622.

[8] - The nonsymmetric Lanczos algorithm and controllability, Systems Control Lett., 16 (1991), pp. 97105.

[9] D. CAlvetti And L. Reichel, On an inverse eigenproblem for Jacobi matrices, Adv. Comput. Math., 11 (1999), pp. 11-20.

[10] M. T. Chu And G. H. Golub, Inverse Eigenvalue Problems: Theory, Algorithms, and Application, Oxford University Press, New York, 2005.

[11] P. A. COJUHARI AND L. P. NIZHNik, Hochstadt inverse eigenvalue problem for Jacobi matrices, J. Math. Anal. Appl., 455 (2017), pp. 439-451.

[12] J. da Providência, N. Bebiano, And J. P. DA Providência, Non-Hermitian Hamiltonians with real spectrum in quantum mechanics, Braz. J. Phys., 41 (2011), pp. 78-85.

[13] R. ERRA AND B. PHILIPPE, On some structured inverse eigenvalue problems, Numer. Algorithms, 15 (1997), pp. 15-35.

[14] W. E. FERGUSON, The construction of Jacobi and periodic Jacobi matrices with prescribed spectra, Math. Comp., 35 (1980), pp. 1203-1220.

[15] G. M. L. Gladwell, Inverse Problems in Vibration, Kluwer, Dordrecht, 2004.

[16] W. Y. HoU AND E. X. JiAnG, A stability analysis of the ( $k$ ) Jacobi matrix inverse eigenvalue problem, Numer. Math. J. Chin. Univ. (Engl. Ser.), 14 (2005), pp. 115-127.

[17] E. X. JiAng, An inverse eigenvalue problem for Jacobi matrices, J. Comput. Math., 21 (2003), pp. 569-584.

[18] H. MIRZAEI, Inverse eigenvalue problem for pseudo-symmetric Jacobi matrices with two spectra, Linear Multilinear Algebra, 66 (2018), pp. 759-768.

[19] C. C. PAIGE, The Computation of Eigenvalues and Eigenvectors of Very Large Sparse Matrices, PhD. Thesis, Institute of Computer Science, University of London, London, 1971.

[20] G. W. Stewart And J. G. Sun, Matrix Perturbation Theory, Academic Press, Boston, 1990.

[21] Q. SU, Inverse spectral problem for pseudo-Jacobi matrices with partial spectral data, J. Comput. Appl. Math., 297 (2016), pp. 1-12.

[22] C. VÖMEL AND J. SLEMONS, Twisted factorization of a banded matrix, BIT, 49 (2009), pp. $433-447$.

[23] Y. WEI AND H. DAI, An inverse eigenvalue problem for Jacobi matrix, Appl. Math. Comput., 251 (2015), pp. 633-642. 
[24] — An inverse eigenvalue problem for the finite element model of a vibrating rod, J. Comput. Appl. Math., 300 (2016), pp. 172-182.

[25] S.-F. XU, A stability analysis of the Jacobi matrix inverse eigenvalue problem, BIT, 33 (1993), pp. 695-702.

[26] - On the Jacobi matrix inverse eigenvalue problem with mixed given data, SIAM J. Matrix Anal. Appl., 17 (1996), pp. 632-639.

[27] — An Introduction to Inverse Algebraic Eigenvalue Problems, Peking University Press, Beijing, Vieweg, Braunschweig, 1998.

[28] W.-R. XU AND G.-L. CHEN, On inverse eigenvalue problems for two kinds of special banded matrices, Filomat, 31 (2017), pp. 371-385.

[29] W.-R. Xu, N. Bebiano, AND G.-L. Chen, An inverse eigenvalue problem for pseudo-Jacobi matrices, Appl. Math. Comput., 346 (2019), pp. 423-435.

[30] - Generalized inverse spectral problem for pseudo-Jacobi matrices with mixed eigendata, Inverse Prob. Sci. Eng., 27 (2019), pp. 773-789. 